IPHT

LPTENS 10/23

\title{
A matrix model for the topological string II The spectral curve and mirror geometry
}

\author{
B. Eynard ${ }^{1}$, A. Kashani-Poor ${ }^{2,3}$, O. Marchal ${ }^{1,4}$ \\ 1 Institut de Physique Théorique, \\ CEA, IPhT, F-91191 Gif-sur-Yvette, France, \\ CNRS, URA 2306, F-91191 Gif-sur-Yvette, France. \\ 2 Institut des Hautes Études Scientifiques \\ Le Bois-Marie, 35, route de Chartres, 91440 Bures-sur-Yvette, France \\ 3 Laboratoire de Physique Théorique de l'École Normale Supérieure, \\ 24 rue Lhomond, 75231 Paris, France \\ ${ }^{4}$ Centre de recherches mathématiques, Université de Montréal \\ C.P. 6128, Succ. centre-ville Montréal, Qué, H3C 3J\%, Canada.
}

\begin{abstract}
In a previous paper, we presented a matrix model reproducing the topological string partition function on an arbitrary given toric Calabi-Yau manifold. Here, we study the spectral curve of our matrix model and thus derive, upon imposing certain minimality assumptions on the spectral curve, the large volume limit of the BKMP "remodeling the B-model" conjecture, the claim that Gromov-Witten invariants of any toric Calabi-Yau 3-fold coincide with the spectral invariants of its mirror curve.
\end{abstract}




\section{Contents}

1 Introduction

2 The fiducial geometry and its mirror 3

2.1 The fiducial geometry . . . . . . . . . . . . . . . . 3

2.2 The mirror of the fiducial geometry . . . . . . . . . . . . . . 4

2.3 The mirror map . . . . . . . . . . . . . . . . . . . . . . . 7

3 Our matrix model 8

4 Generalities on solving matrix models 9

4.1 Introduction to the topological expansion of chain of matrices . . . . . . . 9

4.2 Definition of the general chain of matrices . . . . . . . . . . . . . . . . . 10

4.2.1 The resolvent . . . . . . . . . . . . . . . . 11

4.2.2 The spectral curve of the general chain of matrices . . . . . . . . 11

4.3 Symplectic invariants of a spectral curve . . . . . . . . . . . . . 14

4.3.1 Branchpoints . . . . . . . . . . . . . . . . 14

4.3.2 Bergman kernel . . . . . . . . . . . . . . . . 15

4.3.3 Recursion kernel ................... 15

4.3.4 Topological recursion . . . . . . . . . . . . . . . . . 16

4.3.5 Symplectic invariants . . . . . . . . . . . . . . . 16

5 The spectral curve for the topological string's matrix model 17

5.1 Applying the chain of matrices rules . . . . . . . . . . . . . . . 17

5.2 Symplectic change of functions . . . . . . . . . . . . . . . 21

5.2.1 The arctic circle property . . . . . . . . . . . . . . 21

5.2 .2 Obtaining globally meromorphic functions . . . . . . . . . . 23

5.3 Recovering the mirror curve . . . . . . . . . . . . . . . . . . 25

5.4 Topological expansion and symplectic invariants . . . . . . . . . . . . . 26

5.5 The small $q$ limit and the thickening prescription . . . . . . . . . . . 27

6 The general BKMP conjecture 2

6.1 Flop invariance of toric Gromov-Witten invariants . . . . . . . . . . . . 28

6.2 Proof of flop invariance via mirror symmetry . . . . . . . . . . . . . . 29 29

6.3 The BKMP conjecture . . . . . . . . . . . . . . . . . . . . . 30

7 Conclusion 31

A The matrix model 32 


\section{Introduction}

In a previous paper [1, we presented a matrix model that computes the topological string partition function at large radius on an arbitrary toric Calabi-Yau manifold $\mathfrak{X}$. The goal of this paper is to determine the corresponding spectral curve $\mathcal{S}$.

That the partition function of a matrix model can be recovered to all genus from its spectral curve was first demonstrated in [2]. 3] pushed this formalism further, showing that symplectic invariants $F_{g}(\mathcal{S})$ can be defined for any analytic affine curve $\mathcal{S}$, with no reference to an underlying matrix model. These invariants coincide with the partition function of a matrix model when $\mathcal{S}$ is chosen as the associated spectral curve. The symplectic invariants $F_{g}$ satisfy many properties reminiscent of the topological string partition function [4, 5, 6, 7], motivating Bouchard, Klemm, Mariño, and Pasquetti (BKMP) [8], building on work of Mariño [9], to conjecture that $F_{g}(\mathcal{S})$ in fact coincides with the topological string partition function on the toric Calabi-Yau manifold with mirror curve $\mathcal{S}$. BKMP successfully checked their claim for various examples, at least to low genus. The conjecture was subsequently proved in numerous special cases [10, 11, 12, 13, 14, 15].

Bouchard and Mariño [16] noticed that an infinite framing limit of the BKMP conjecture for the framed vertex, $\mathfrak{X}=\mathbb{C}^{3}$, implies a conjecture for the computation of Hurwitz numbers, namely that the Hurwitz numbers of genus $g$ are the symplectic invariants of genus $g$ for the Lambert spectral curve $e^{x}=y e^{-y}$. This conjecture was proved recently by a generalization of [10] using a matrix model for summing over partitions [17], and also by a direct combinatorial method [18]. Matrix models and the BKMP conjecture related to toric Calabi-Yau geometries arising from the triangulation of a strip were recently studied in [19].

In this paper, we derive the large radius limit of the BKMP conjecture for arbitrary toric Calabi-Yau manifolds, but with one caveat: to determine the spectral curve of our matrix model, we must make several minimality assumptions along the way. To elevate our results to a rigorous proof of the BKMP conjecture, one needs to establish a uniqueness result underlying our prescription for finding the spectral curve to justify these minimal choices. Such a uniqueness result does not exist to date.

Recall that in [1, we first compute the topological string partition function on a toric Calabi-Yau geometry $\mathfrak{X}_{0}$ which we refer to as fiducial. We then present a matrix model which reproduces this partition function. Flops and limits in the Kähler cone relate $\mathfrak{X}_{0}$ to an arbitrary toric Calabi-Yau 3-fold. As we can follow the action of these operations on the partition function, we thus arrive at a matrix model for the topological string on any toric Calabi-Yau 3-folds. Here, we follow the analogous strategy, by first computing the spectral curve of the matrix model associated to $\mathfrak{X}_{0}$, and then studying the action of flops and limits on this curve.

The plan of the paper is as follows. In section 2, we introduce the fiducial geometry $\mathfrak{X}_{0}$ and its mirror. The matrix model reproducing the partition function on $\mathfrak{X}_{0}$, as derived in [1], is a chain of matrices matrix model. It is summarized in section 3 and appendix A. We review general aspects of this class of matrix models and their solutions in section 
4. In section 5, we determine a spectral curve which satisfies all specifications outlined in section 4, and demonstrate that it coincides, up to symplectic transformations, with the B-model mirror of the fiducial geometry. While in our experience with simpler models, the conditions of section 4 on the spectral curve specify it uniquely, we lack a proof of this uniqueness property. We thus provide additional consistency arguments for our proposal for the spectral curve in section 5.5. Flops and limits in the Kähler cone relate the fiducial to an arbitrary toric Calabi-Yau manifold. Following the action of these operations on both sides of the conjecture in section 6 completes the argument yielding the BKMP conjecture for arbitrary toric Calabi-Yau manifolds in the large radius limit. We conclude by discussing possible avenues along this work can be extended.

\section{The fiducial geometry and its mirror}

\subsection{The fiducial geometry}

In [1, we derived a matrix model reproducing the topological string partition function on the toric Calabi-Yau geometry $\mathfrak{X}_{0}$ whose toric fan is depicted in figure 1 . We refer to $\mathfrak{X}_{0}$ as our fiducial geometry; we will obtain the partition function on an arbitrary toric Calabi-Yau manifolds by considering flops and limits of $\mathfrak{X}_{0}$.

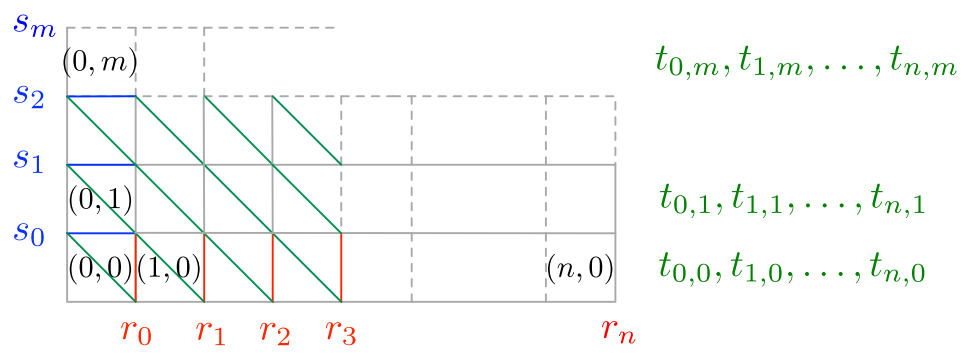

Figure 1: Fiducial geometry $\mathfrak{X}_{0}$ with boxes numbered and choice of basis of $H_{2}\left(\mathfrak{X}_{0}, \mathbb{Z}\right)$.

We have indicated a basis of $H_{2}\left(\mathfrak{X}_{0}, \mathbb{Z}\right)$ in figure 1 . Applying the labeling scheme introduced in figure 2, the curve classes of our geometry are expressed in this basis as follows,

$$
\begin{aligned}
r_{i, j} & =r_{i}+\sum_{k=1}^{j}\left(t_{i+1, k-1}-t_{i, k}\right) \\
s_{i, j} & =s_{j}+\sum_{k=1}^{i}\left(t_{k-1, j+1}-t_{k, j}\right) .
\end{aligned}
$$

It proves convenient to express these classes as differences of what we will refer to as $a$-parameters [1], defined via

$$
t_{i, j}=a_{i, j}-a_{i, j+1} \quad, \quad r_{i, j}=a_{i, j+1}-a_{i+1, j} .
$$




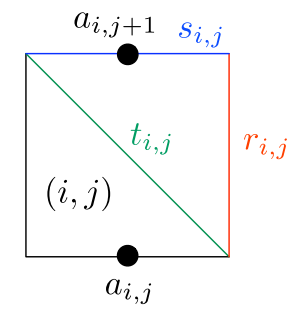

Figure 2: Labeling curve classes, and introducing $a$-parameters.

\subsection{The mirror of the fiducial geometry}

The Hori-Vafa prescription [20] allows us to assign a mirror curve to a toric Calabi-Yau manifold. Each torically invariant divisor, corresponding to a 1-cone $\rho \in \Sigma(1)$, is mapped to a $\mathbb{C}^{*}$ variable $e^{-Y_{\rho}}$. These are constrained by the equation

$$
\sum_{\rho \in \Sigma(1)} e^{-Y_{\rho}}=0
$$

Relations between the 1-cones, as captured by the lattice $\Lambda_{h}$ introduced in section (2.1) of [1], map to relations between these variables: for $\sigma \in \Sigma(2)$,

$$
\sum_{\rho \in \Sigma(1)} \lambda_{\rho}(\sigma) Y_{\rho}=W_{\sigma} .
$$

The $W_{\sigma}$ are complex structure parameters of the mirror geometry, related to the Kähler parameters $w_{\sigma}=r_{i, j}, s_{i, j}, \ldots$ introduced in the previous subsection via the mirror map, as we will explain in the next subsection.

The Hori-Vafa prescription gives rise to the following mirror curve $\mathcal{C}_{\mathfrak{X}_{0}}$ of our fiducial geometry $\mathfrak{X}_{0}$,

$$
\sum_{i=0}^{n+1} \sum_{j=0}^{m+1} x_{i, j}=0 .
$$

We have here labeled the 1 -cones by coordinates $(i, j)$, beginning with $(0,0)$ for the cone $(0,0,1)$ in the bottom left corner of box $(0,0)$ as labeled in figure 1 , and introduced the notation

$$
x_{i, j}=e^{-Y_{i, j}} .
$$

Eliminating dependent variables by invoking (2.1) yields an equation of the form

$$
\sum_{i=0}^{n+1} \sum_{j=0}^{m+1} c_{i, j} z_{i, j}=0 .
$$

Here,

$$
z_{i, j}=x_{0}^{1-i-j} x_{1}^{i} x_{2}^{j}
$$


where we have defined

$$
x_{0}=x_{0,0}, x_{1}=x_{1,0}, x_{2}=x_{0,1} .
$$

$\left(x_{0}: x_{1}: x_{2}\right)$ define homogeneous coordinates on $\mathbb{C P}^{2}$. The form of the equation is independent of the choice of triangulation of the toric diagram. What does depend on this choice are the coefficients $c_{i, j}$. It is not hard to write these down for the fiducial geometry $\mathfrak{X}_{0}$ with the choice of basis for $H_{2}\left(\mathfrak{X}_{0}, \mathbb{Z}\right)$ indicated in figure 1 . Explicitly, the relations between the coordinates of the mirror curve 2.2 are

$$
x_{i, 0}=\frac{x_{i-1,0} x_{i-1,1}}{x_{i-2,1}} e^{R_{i-2}}, \quad x_{0, j}=\frac{x_{0, j-1} x_{1, j-1}}{x_{1, j-2}} e^{S_{j-2}}, \quad x_{i, j}=\frac{x_{i-1, j} x_{i, j-1}}{x_{i-1, j-1}} e^{T_{i-1, j-1}} .
$$

Solving in terms of $x_{0}, x_{1}, x_{2}$ yields the coefficients $c_{0,0}=c_{0,1}=c_{1,0}=1$,

$$
\begin{aligned}
& c_{i, 0}=\exp \left[\sum_{k=1}^{i-1}(i-k)\left(R_{k-1}+T_{k-1,0}\right)\right], \\
& c_{0, j}=\exp \left[\sum_{l=1}^{j-1}(j-l)\left(S_{l-1}+T_{0, l-1}\right)\right],
\end{aligned}
$$

and for $i, j>0$

$c_{i, j}=\exp \left[(i+j-1) T_{0,0}+\sum_{k=1}^{i-1}(i-k)\left(R_{k-1}+T_{k, 0}\right)+\sum_{l=1}^{j-1}(j-l)\left(S_{l-1}+T_{0, l}\right)+\sum_{k=1}^{i-1} \sum_{l=1}^{j-1} T_{k, l}\right]$.

Note that the number of coefficients $c_{i, j}$, up to an overall rescaling, is equal to the number of independent curve classes $r_{i}, s_{j}, t_{i, j}$.

In [21], the thickening prescription was put forth for determining the genus and number of punctures of the mirror curve: one is to thicken the web diagram of the original geometry to obtain the Riemann surface of the mirror geometry. The procedure is illustrated in figure 3. We will now verify this procedure by studying the curve (2.3) explicitly.

Let's consider the curve (2.3) for a single strip (i.e. $n=0$ ) of length $m+1$,

$$
\begin{array}{r}
x_{0}^{m+2}+x_{0}^{m+1} x_{1}+x_{0}^{m+1} x_{2}+c_{1,1} x_{0}^{m} x_{1} x_{2}+c_{2,0} x_{0}^{m} x_{1}^{2}+c_{2,1} x_{0}^{m-1} x_{1}^{2} x_{2}+c_{3,0} x_{0}^{m-1} x_{1}^{3}+\ldots \\
+c_{m+1,0} x_{0} x_{1}^{m+1}+c_{m+1,1} x_{1}^{m+1} x_{2}=0 .
\end{array}
$$

Note that the equation is of degree $m+2$, but the point $(0: 0: 1)$ is an $m+1$-tuple point. By choosing the coefficients to be generic, we can arrange for this singular point to be ordinary. The genus formula then yields

$$
\mathfrak{g}=\frac{(d-1)(d-2)}{2}-\frac{m(m+1)}{2}=0
$$



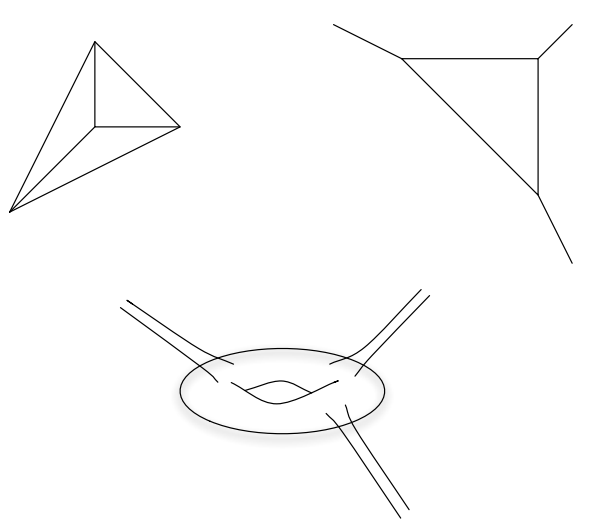

Figure 3: Example of the thickening prescription: depicted are the fan for $\mathcal{O}(-3) \rightarrow \mathbb{P}^{2}$, the corresponding web diagram, and the mirror curve obtained via the thickening prescription.

In terms of the physical variables $Y_{i}$, any point on the curve with a vanishing homogeneous coordinate corresponds to a puncture. The punctures on the curve (2.5) thus lie at

$$
\begin{array}{rll}
(0: 0: 1) & : & m+1 \\
(0: 1: 0) & : & 1 \\
\left(1: x_{1}^{i}: 0\right) & : & m+1 \\
(1: 0:-1) & : & 1,
\end{array}
$$

where $x_{1}^{i}, i=1, \ldots, m+1$, are the solutions of the equation

$$
1+x_{1}+\sum_{j=1}^{m} d_{i} x_{1}^{i+1}=0 .
$$

Note that we reproduce the $2 m+4$ punctures expected from the thickening prescription of the toric diagram.

For the general case parametrized by $(m, n)$, the degree of the curve is $d=m+n+2$, and we have an ordinary $m+1$-tuple point at $(0: 0: 1)$ and an ordinary $n+1$-tuple point at $(0: 1: 0)$. The genus formula now yields

$$
g=\frac{(m+n)(m+n+1)}{2}-\frac{m(m+1)}{2}-\frac{n(n+1)}{2}=m n
$$

The punctures lie at

$$
\begin{array}{rll}
(0: 0: 1) & : & m+1 \\
(0: 1: 0) & : & n+1 \\
\left(1: x_{1}^{i}: 0\right) & : & m+1 \\
\left(1: 0: x_{2}^{j}\right) & : & n+1
\end{array}
$$

with $x_{1}^{i}$ the roots of $\sum_{i=0}^{m+1} c_{i, 0} x_{1}^{i}=0$ and $x_{2}^{j}$ the roots of $\sum_{j=0}^{n+1} c_{0, j} x_{2}^{j}=0$. Again, we see that we reproduce the thickening prescription. 


\subsection{The mirror map}

Above, we have distinguished between Kähler ( $A$-model) parameters $w_{\sigma}$ and complex structure ( $B$-model) parameters $W_{\sigma}$. At large radius/complex structure, these are identified between mirror pairs, but this identification is corrected by the so-called mirror map ${ }^{1}$

$$
W_{\sigma}=w_{\sigma}+\mathcal{O}\left(e^{-w_{\sigma}}\right) .
$$

The exponentials of the parameters $W_{\sigma}$ appear as coefficients in the equation defining the mirror curve. They are global coordinates on the complex structure moduli space of the mirror curve. To compare expressions obtained in the A-model to those obtained in the B-model, all expressions are conventionally expressed in terms of flat coordinates $w_{\sigma}$. On the A-model side, these coordinates enter (in exponentiated form denoted generically as $Q_{\alpha, \beta}$ below) in the definition of the topological vertex. On the B-model side, they arise as the appropriate periods of a meromorphic one-form $\lambda$, defined in terms of the affine variables $x=\frac{x_{1}}{x_{0}}, y=\frac{x_{2}}{x_{0}}$ in the patch $x_{0} \neq 0$ of the curve 2.3 as

$$
\lambda=\log y \frac{d x}{x} .
$$

By calculating these periods as a function of the coefficients defining the mirror curve, we obtain the mirror map (2.6).

The coordinates $w_{\sigma}$ are not globally defined functions on the complex structure moduli space. In the slightly clearer compact setting, this is due to the fact that the symplectic basis $\left\{\alpha_{A}, \beta^{A}\right\}$ of $H^{3}(\mathfrak{X}, \mathbb{Z})$ in which we expand $\Omega$ (the compact analogue of the meromorphic 1-form $\lambda$ introduced above) such that the coefficients of $\alpha_{A}$ furnish our (local) coordinate system of the complex structure moduli space, undergo monodromy when transported around a singularity in moduli space $2^{2}$ A good choice of coordinates in the vicinity of a singular divisor $D$ hence involves a choice of basis forms that are invariant under monodromy around that divisor.

\footnotetext{
${ }^{1}$ One could take exception to this nomenclature, arguing that the parameters $W_{\sigma}$ are the geometric parameters on both sides of the mirror, and refer to the $w_{\sigma}$ as the instanton or quantum corrected parameters. In such conventions, the curve classes in the various toric diagrams should be labeled by upper case letters.

${ }^{2}$ Note that the symplectic basis makes no reference to complex structure, one might hence be led to believe that a global choice (i.e. one valid for any choice of complex structure) should be possible. This is not so. We consider the family $\pi: \mathcal{X} \rightarrow \mathcal{S}$, with $\mathcal{S}$ the complex structure moduli space. The fiber over each point $w \in \mathcal{S}, \pi^{-1} w=X_{w}$, is the Calabi-Yau manifold with the respective complex structure. $H^{n}\left(X_{w}, \mathbb{C}\right)$ fit together to form a vector bundle $\mathcal{F}_{0}$ over $\mathcal{S}$, with a canonical flat connection, the GaussManin connection. Using this connection, we can parallel transport a symplectic basis of $H^{3}\left(X_{w}, \mathbb{C}\right)$ along a curve in $\mathcal{S}$. As $\mathcal{S}$ is not generically simply connected (due to the existence of degeneration points of the geometry), this transport may exhibit monodromy. Note that $\Omega$ can be defined as the section of a sheaf in the Hodge filtration of $H^{3}$ which extends to the singular divisor, hence is single valued. The monodromy in our choice of flat coordinates is therefore entirely due to the choice of symplectic basis.
} 


\section{Our matrix model}

We derived a chain of matrices matrix model that reproduces the topological string partition function on $\mathfrak{X}_{0}$ in [1]. For $\mathfrak{X}_{0}$ of size $(n+1) \times(m+1)$, as depicted in figure 1 , it is given by

$$
\begin{aligned}
Z_{\mathrm{MM}}\left(\vec{Q}, g_{s}, \vec{\alpha}_{m+1}, \vec{\alpha}_{0}^{T}\right)= & \Delta\left(X\left(\vec{\alpha}_{m+1}\right)\right) \Delta\left(X\left(\vec{\alpha}_{0}\right)\right) \prod_{i=0}^{m+1} \int_{H_{N}\left(\Gamma_{i}\right)} d M_{i} \prod_{i=1}^{m+1} \int_{H_{N}\left(\mathbb{R}_{+}\right)} d R_{i} \\
& \prod_{i=1}^{m} e^{\frac{-1}{g_{s}} \operatorname{tr}\left[V_{\vec{a}_{i}}\left(M_{i}\right)-V_{\vec{a}_{i-1}}\left(M_{i}\right)\right]} \prod_{i=1}^{m} e^{\frac{-1}{g_{s}} \operatorname{tr}\left[V_{\vec{a}_{i-1}}\left(M_{i-1}\right)-V_{\vec{a}_{i}}\left(M_{i-1}\right)\right]} \\
& \prod_{i=1}^{m+1} e^{\frac{1}{g_{s}} \operatorname{tr}\left(M_{i}-M_{i-1}\right) R_{i}} \prod_{i=1}^{m} e^{\left(S_{i}+\frac{i \pi}{g_{s}}\right) \operatorname{tr} \ln M_{i}} \\
& e^{\operatorname{tr} \ln f_{0}\left(M_{0}\right)} e^{\operatorname{tr} \ln f_{m+1}\left(M_{m+1}\right)} \prod_{i=1}^{m} e^{\operatorname{tr} \ln f_{i}\left(M_{i}\right)} .
\end{aligned}
$$

We give the explicit expressions for the various functions entering in this definition in appendix A. Here, we briefly explain some of its general features.

The matrix model (3.1) is designed to reproduce the topological string partition function on the toric Calabi-Yau manifold $\mathfrak{X}_{0}$ as computed using the topological vertex [22]. Recall that in this formalism, the dual web diagram to the toric diagram underlying the geometry is decomposed into trivalent vertices. Each such vertex contributes a factor $C\left(\alpha_{i}, \alpha_{j}, \alpha_{k}\right)$ [22], where $\alpha_{i}$ denote Young tableaux (partitions) of arbitrary size, one associated to each leg of the vertex. Legs of different vertices are glued by matching these Young tableaux and summing over them with appropriate weight.

Aside from the coupling constant $g_{s}$ and Kähler parameters of the geometry, denoted collectively as $\vec{Q}$, the matrix model $(3.1)$ depends on partitions $\vec{\alpha}_{0}, \vec{\alpha}_{m+1}$ associated to the outer legs of the web diagram, which we choose to be trivial in this paper. The two classes of integrals $d R_{i}$ and $d M_{i}$ correspond to the two steps in which the topological string partition function on the fiducial geometry $\mathfrak{X}_{0}$ can be evaluated: First, the geometry can be decomposed into $m+1$ horizontal strips, with partitions $\alpha_{j, i+1}$ and $\alpha_{j, i}$ associated to the upper and lower outer legs of the associated strip web diagram. $j=0, \ldots, n$ counts the boxes in figure 1 in the horizontal direction, $i=0, \ldots, m+1$ is essentially the strip index. Each such strip has a $d R_{i}$ integration associated to it. The partition function on such strips was calculated in [23]. Following [12], we introduce two matrices $M_{i}, M_{i+1}$ per strip. Their eigenvalues encode the partitions $\alpha_{j, i}$ and $\alpha_{j, i+1}$ for all $j$. To work with finite size matrices, we introduce a cut-off $d$ on the number of rows of the Young tableaux we sum over. As we argue in section 5.2.1, our matrix model depends on $d$ only non-perturbatively. The strip partition function is essentially given by the Cauchy determinant of the two matrices $M_{i}, M_{i+1}$ [1], and the $d R_{i}$ integrals are the associated Laplace transforms. Gluing the strips together involves summing over the partitions $\alpha_{j, i}$. This step is implemented by the $d M_{i}$ integrations. To obtain a discrete sum over partitions 
from integration, we introduce functions $f_{i}\left(M_{i}\right)$ with integrally spaced poles. Integrating $M_{i}$ along appropriate contours then yields the sum over partitions as a sum over residues, the potentials $V_{\vec{a}_{i}}$ chosen to provide the proper weight per partition.

\section{Generalities on solving matrix models}

\subsection{Introduction to the topological expansion of chain of matri- ces}

Chain of matrices matrix models have been extensively studied (see Mehta's book [24] and the review article [25]), and the computation of their topological expansion was performed recently in [26, 27].

The solution provided in [27] is based on the computation of the spectral curve $\mathcal{S}_{\mathrm{MM}}$ of the matrix model. In [27, 26], only the case of potentials whose derivatives are rational functions is considered, and similarly to the one matrix model, the planar ${ }^{3}$ expectation value of the resolvent of the first matrix of the chain is shown to satisfy an algebraic equation. The spectral curve is defined to be the solution locus of this equation. A general recipe is provided in [26, 27] to obtain the spectral curve from algebraic equations and analyticity properties related to rational potentials and integration contours. Here, our potentials contain logs of $g$-functions. As they are not rational, we will have to present a slight extension of the recipe of [27] in section 4.2.2. This extension from rational potentials to analytical potentials, although not published, is straightforward, and the derivation of these results will appear in [28]. In some sense, the derivative of $\ln g(x)$ can be viewed as a rational function with an infinite number of simple poles, i.e. as a limit of a rational function. More precisely, as an expansion in powers of $q$, to each order, it is a rational function. Since the spectral curve can be described by local properties, independent of the number of poles, one can take the limit of the recipe of [26, 27]. This is what we shall do in section 4.2 .2 below.

Having found the spectral curve $\mathcal{S}_{\mathrm{MM}}$ of the matrix model, we will compute its symplectic invariants

$$
F_{g}\left(\mathcal{S}_{\mathrm{MM}}\right), \quad g=0,1,2,3, \ldots
$$

Symplectic invariants $F_{g}(\mathcal{S})$ can be computed for any analytical plane curve $\mathcal{S}$, and thus in particular for $\mathcal{S}=\mathcal{S}_{\mathrm{MM}}$. For a general $\mathcal{S}$ they were first introduced in [3], as a generalization of the solution of matrix models loop equations of [2]. Their definition

\footnotetext{
${ }^{3}$ For matrix models with $N$-independent polynomial potentials whose $g_{s}$ dependence is given by an overall prefactor, the planar limit coincides with the large $N$ limit, but this correspondence can fail if the potential or the integration contours have a non-trivial $N$ or $g_{s}$ dependence. The planar limit is defined by keeping only planar graphs in the Feynman graph perturbative expansion around an extremum of the potential. However, it is helpful to have in mind the intuitive picture that the planar limit is similar to a large $N$ limit.
} 
is algebraic and involves computation of residues at branch points of $\mathcal{S}$. We recall the definition below in section 4.3 .

\subsection{Definition of the general chain of matrices}

We consider chain of matrices matrix models of the form

$$
Z=\int_{\mathcal{E}} d M_{1} \ldots d M_{L} e^{-\frac{1}{g_{s}} \operatorname{Tr} \sum_{i=1}^{L} V_{i}\left(M_{i}\right)} e^{\frac{1}{g_{s}} \operatorname{Tr} \sum_{i=1}^{L-1} c_{i} M_{i} M_{i+1}} .
$$

Note that aside from the potentials $V_{i}\left(M_{i}\right)$, the only interactions are between nearest neighbors, whence the name "chain of matrices." Chain of matrices matrix models can be solved when the interaction terms between different matrices are of the form $\operatorname{Tr} M_{i} M_{i+1}$, as is the case here.

$\mathcal{E}$ can be any ensemble of $L$ normal matrices of size $N \times N$, i.e. a submanifold of $\mathbb{C}^{L N^{2}}$ of real dimension $L N^{2}$, such that the integral is convergent. $\mathcal{E}$ can be many things; for a chain of matrices model, it is characterized by the contours on which eigenvalues of the various normal matrices are integrated (see [29] for the 2-matrix model case). For (4.1) to have a topological expansion, $\mathcal{E}$ must be a so-called steepest descent ensemble (see [30], section 5.5). For a generic ensemble $\mathcal{E}$ which would not be steepest descent, $\ln Z$ would be an oscillating function of $1 / g_{s}$, and no small $g_{s}$ expansion would exist, see [31].

The matrix model introduced in [1] and reproduced in section 3 was defined to reproduce the topological string partition function, which is defined as a formal series in $g_{s}$, and therefore has a topological expansion by construction.

An ensemble $\mathcal{E}$ is characterized by filling fractions $n_{j, i}$,

$$
\mathcal{E}=\prod_{i=1}^{L} \mathcal{E}_{i} \quad, \quad \mathcal{E}_{i}=H_{N}\left(\gamma_{1, i}^{n_{1, i}} \times \gamma_{2, i}^{n_{2, i}} \times \cdots \times \gamma_{k_{i}, i}^{n_{k_{i}, i}}\right)
$$

where $H_{N}\left(\gamma_{1}^{n_{1}} \times \cdots \times \gamma_{k}^{n_{k}}\right)$ is the set of normal matrices with $n_{1}$ eigenvalues on path $\gamma_{1}$, $n_{2}$ eigenvalues on path $\gamma_{2}, \ldots, n_{k}$ eigenvalues on path $\gamma_{k}$.

As the filling fractions $n_{j, i}$ must satisfy the relation

$$
\sum_{j=1}^{k_{i}} n_{j, i}=N
$$

for all $i$, only $\sum_{i}\left(k_{i}-1\right)$ of them are independent.

We also allow some paths $\gamma_{j, i}$ to have endpoints where $e^{-\operatorname{Tr} \sum_{i=1}^{L}\left(V_{i}\left(M_{i}\right)-M_{i} M_{i+1}\right)} \neq 0-$ indeed, in our matrix model, the matrices $R_{i}$ are integrated on $H\left(\mathbb{R}_{+}^{N}\right)$. 


\subsubsection{The resolvent}

The spectral curve encodes all $W_{i}(x)$, the planar limits (see footnote 3 ) of the resolvents of the matrices $M_{i}$,

$$
W_{i}(x)=g_{s}\left\langle\operatorname{tr} \frac{1}{x-M_{i}}\right\rangle_{\text {planar }},
$$

see equation (4.5) below. The respective $W_{i}$ can be expressed as the Stieljes transform

$$
W_{i}(x)=\int \frac{\rho_{i}\left(x^{\prime}\right) d x^{\prime}}{x-x^{\prime}}
$$

of the planar expectation value of the eigenvalue density $\rho_{i}(x)$ of the matrix $M_{i}$,

$$
\rho_{i}(x)=g_{s}\left\langle\operatorname{tr} \delta\left(x-M_{i}\right)\right\rangle_{\text {planar }} .
$$

By general properties of Stieljes transforms, singularities of $W_{i}(x)$ coincide with the support of the distribution $\rho_{i}(x) d x$ :

- Simple poles of $W_{i}(x)$ correspond to delta distributions i.e. isolated eigenvalues.

- Multiple poles correspond to higher derivatives of delta distributions.

- Cuts correspond to finite densities, the density being the discontinuity of $W_{i}(x)$ along the cut,

$$
\rho_{i}(x)=\frac{1}{2 i \pi}\left(W_{i}(x-i 0)-W_{i}(x+i 0)\right) .
$$

In particular, cuts emerging from algebraic singularities (generically square root singularities) correspond to densities vanishing algebraically (generically as square roots) at the endpoints of the cut. Cuts emerging from logarithmic singularities correspond to constant densities.

\subsubsection{The spectral curve of the general chain of matrices}

When all $V_{i}^{\prime}$ are rational, the spectral curve was found in [26, 27], and it is algebraic. We present here a generalization of this result to more general potentials. The derivations of these results will appear in [28].

The spectral curve can be obtained by the following procedure:

1. Consider a compact Riemann surface $\mathcal{C}$ of genus

$$
\mathfrak{g}=\sum_{i=1}^{L}\left(k_{i}-1\right),
$$

where $k_{i}$ denotes the number of cuts of the $i$-th matrix, as implicitly defined in 4.2 .

2. Look for $L+2$ functions on $\mathcal{C}$,

$$
x_{0}(z), x_{1}(z), x_{2}(z), \ldots, x_{L}(z), x_{L+1}(z): \mathcal{C} \rightarrow \mathbb{C P}^{1} .
$$


The $x_{i}$ are to be holomorphic away from points $z \in \mathcal{C}$ at which $V_{i-1}^{\prime}\left(x_{i-1}\right)$ or $V_{i+1}^{\prime}\left(x_{i+1}\right)$ become singular, and satisfy the functional relations

$$
c_{i-1} x_{i-1}(z)+c_{i} x_{i+1}(z)=V_{i}^{\prime}\left(x_{i}(z)\right) .
$$

Recall that the $c_{i}$ are the coefficients of the interaction potentials in (4.1). We have set $c_{0}=c_{L}=1$.

For each $i=1, \ldots, L$, the Riemann surface $\mathcal{C}$ can be realized as a branched covering of $\mathbb{C P}^{1}$ by the projection $x_{i}: \mathcal{C} \rightarrow \mathbb{C P}^{1}$. A choice of branched covering is not unique: the choice consists in the set of cuts connecting branch points (recall that these are points at which $\left.d x_{i}(z)=0\right)$. We will determine an appropriate covering below in step 4.

3. If some path $\gamma_{j, i}$ has an endpoint $a$ (called "hard edge" in the matrix model literature, see [32]), then choose a pre-image $a_{i} \in x_{i}^{-1}(a)$ and require

$$
d x_{i}\left(a_{i}\right)=0 \quad \text { and } \quad x_{i-1}(z) \text { has a simple pole at } z=a_{i} .
$$

The topological recursion is proved in [27] without hard edges, but it is not difficult to see, by mixing the results of [32, 33] and [27, that the topological recursion continues to hold in the presence of hard edges. The proof will appear in a forthcoming publication [28]. Here, we shall assume that it holds.

4. Choose some contours $\widehat{\mathcal{A}}_{j, i}, j=1, \ldots, k_{i}$ in $\mathbb{C P}^{1}$, such that each $\widehat{\mathcal{A}}_{j, i}$ surrounds all points of the contour $\gamma_{j, i}$ (related to the matrix ensemble $\mathcal{E}_{i}$ defined in (4.2)) in the clockwise direction and no other contour $\gamma_{j^{\prime}, i}$. For $x \in \mathbb{C P}^{1}$ not enclosed in the contours $\widehat{\mathcal{A}}_{j, i}, j=1, \ldots, k_{i}$, and given a connected component $\mathcal{A}_{j, i}$ of the pre-image of the contour $\widehat{\mathcal{A}}_{j, i}$ under $x_{i}$,

$$
\mathcal{A}_{j, i} \subset x_{i}^{-1}\left(\widehat{\mathcal{A}}_{j, i}\right)
$$

define the function

$$
W_{i}(x)=\frac{c_{i-1}}{2 i \pi} \sum_{j=1}^{k_{i}} \oint_{\mathcal{A}_{j, i}} \frac{x_{i-1}(z) d x_{i}(z)}{x-x_{i}(z)} .
$$

Generalizing [26] to non-polynomial potentials, we claim that a choice of $\mathcal{A}_{j, i}$ exists such that $W_{i}(x)$ is the planar limit of the resolvent of the matrix $M_{i}$. In the following, it is this choice that will be referred to as $\mathcal{A}_{j, i}$.

Notice that not all $\mathcal{A}_{j, i}$ will be homologically independent on $\mathcal{C}$. We require that we have $\mathfrak{g}=\sum_{i=1}^{L}\left(k_{i}-1\right)$ homologically independent $\mathcal{A}_{j, i}$ 's, which coincides with the genus of $\mathcal{C}$. As a condition on the choice of branched covering, we impose that $\mathcal{A}_{j, i}$ and $a_{i}$ lie on the same sheet of $x_{i}$. This condition, in our experience, uniquely fixes this choice. We will assume that this is the case. We refer to the sheet of $x_{i}$ containing $\mathcal{A}_{j, i}$ and $a_{i}$ as the physical sheet for $x_{i}$. 
5. In accord with (4.3), we consider the discontinuity of $W_{i}(x)$ along the $j$-th cut. It is given by

$$
\begin{aligned}
\underset{j}{\operatorname{Disc}} W_{i}(x) & =\frac{1}{2 \pi i}\left(W_{i}\left(x_{+}\right)-W_{i}\left(x_{-}\right)\right) \\
& =\frac{1}{2 \pi i} c_{i-1} \operatorname{Disc}_{j} x_{i-1},
\end{aligned}
$$

as we explain in figure 5 .

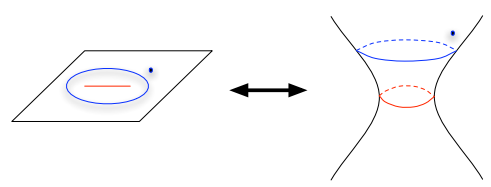

Figure 4: The integration contour $\widehat{\mathcal{A}}_{j, i}$ on the $x$-plane, and its image $\mathcal{A}_{j, i}$ on $\mathcal{C}$.

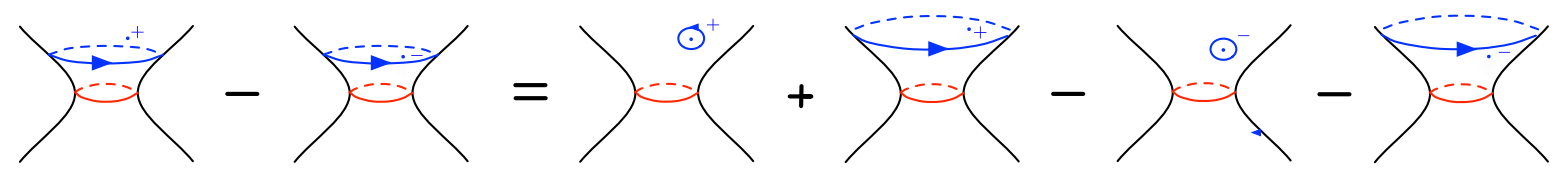

Figure 5: The preimage of the points $x_{+}$and $x_{-}$of 4.6 are depicted as dots in the above diagram, $\widehat{\mathcal{A}}_{j, i}$ is given by the blue contour, and the preimage of the cut is drawn in red. To take the limit $x_{+} \rightarrow x_{-}$, one must first shift the contours. The second and fourth term on the RHS of the above diagrammatic equation then cancel, yielding the RHS of 4.6.

The definition (4.2) of the matrix ensemble $\mathcal{E}_{i}$ is the condition that there are $n_{j, i}$ eigenvalues of $\bar{M}_{i}$ on the contour $\gamma_{j, i}$, hence corresponds to imposing the filling fraction conditions

$$
\frac{1}{2 \pi i} \oint_{\mathcal{A}_{j, i}} c_{i-1} x_{i-1} d x_{i}=g_{s} n_{j, i}
$$

for $i=1, \ldots, L, j=1, \ldots, k_{i}$.

In our experience, the conditions enumerated above have a unique solution and define a unique spectral curve. As emphasized in the introduction, a formal uniqueness proof is however still lacking.

The spectral curve is defined as the data of the Riemann surface $\mathcal{C}$, and the two functions $x_{1}(z)$ and $x_{2}(z)$,

$$
\mathcal{S}_{M M}=\left(\mathcal{C}, x_{1}, x_{2}\right) .
$$




\subsection{Symplectic invariants of a spectral curve}

Once we have found the spectral curve $\mathcal{S}_{M M}$ of our matrix model, we can compute the coefficients $F_{g}$ in the topological expansion of its partition function,

$$
\ln Z=\sum_{g=0}^{\infty} g_{s}^{2 g-2} F_{g},
$$

by computing the symplectic invariants of this curve,

$$
F_{g}=F_{g}\left(\mathcal{S}_{M M}\right)
$$

following [27].

Let us recall the definition of these invariants for an arbitrary spectral curve $\mathcal{S}$.

Let $\mathcal{S}=(\mathcal{C}, x, y)$ be a spectral curve, comprised of the data of a Riemann surface $\mathcal{C}$ and two functions $x(z), y(z): \mathcal{C} \rightarrow \mathbb{C}$, meromorphic on $\mathcal{C}$ away from a finite set of points (we wish to allow logarithms). ${ }^{4}$ We will assume that $d x$ is a meromorphic form on all of $\mathcal{C}$.

\subsubsection{Branchpoints}

Let $a_{i}$ be the branch points of the function $x$,

$$
d x\left(a_{i}\right)=0 .
$$

We assume that all branch points are simple, i.e. that $d x$ has a simple zero at $a_{i}$. This implies that in the vicinity of $a_{i}$, the map $x$ is $2: 1$. We introduce the notation $\bar{z} \neq z$ such that

$$
x(\bar{z})=x(z) .
$$

$\bar{z}$ is called the conjugate point to $z$, and it is defined only in the vicinity of branch points, as depicted in figure 6 .

We also require that the branch points of $x$ and $y$ do not coincide, such that $d y\left(a_{i}\right) \neq 0$ and $y(z)$ therefore has a square-root branchcut as a function of $x$ at $x\left(a_{i}\right)$. If $y$ is finite at $a_{i}$, its local behavior is hence given by

$$
y(z) \sim y\left(a_{i}\right)+C_{i} \sqrt{x(z)-x\left(a_{i}\right)} .
$$

If $a_{i}$ corresponds to a hard edge, we require $y$ to have a pole here. Its local behavior is hence given by

$$
y(z) \sim \frac{C_{i}}{\sqrt{x(z)-x\left(a_{i}\right)}} .
$$

\footnotetext{
${ }^{4}$ In fact, the most general setting in which this formalism is valid has not yet been established. We will state it within the generality we need here i.e. we assume that $d x$ is meromorphic forms on $\mathcal{C}$ (this allows $x$ and $y$ to have logarithms).
} 


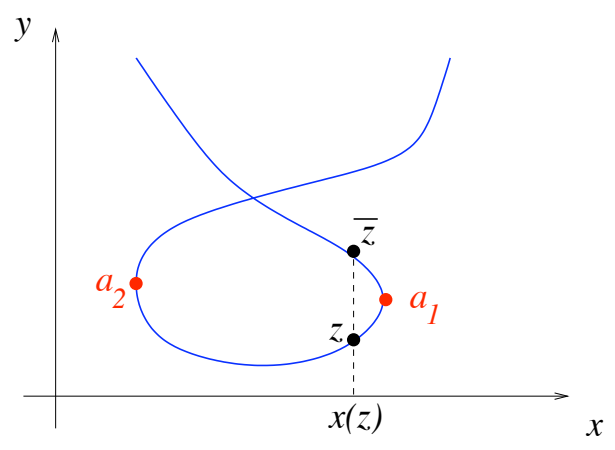

Figure 6: At a regular branch point $a \in \mathcal{C}$ of $x, y$ as a function of $x$ has a branchcut $y \sim y(a)+$ $C \sqrt{x-x(a)}$. If $z$ is a point on one branch near $a$, we call $\bar{z}$ the conjugate point on the other branch; it has the same $x$ projection, $x(\bar{z})=x(z)$. Notice that $\bar{z}$ is defined only locally near branch points. If we follow $z$ from $a_{1}$ to $a_{2}, \bar{z}$ may have to jump from one branch to another.

\subsubsection{Bergman kernel}

On a curve $\mathcal{C}$, there exists a unique symmetric 2 -form $B\left(z_{1}, z_{2}\right)$ with a double pole on the diagonal $z_{1}=z_{2}$ and no other poles, with the following normalization on $\mathcal{A}$-cycles,

$$
\oint_{z_{2} \in \mathcal{A}_{j, i}} B\left(z_{1}, z_{2}\right)=0
$$

In any local coordinate near $z_{1}=z_{2}$, one has

$$
B\left(z_{1}, z_{2}\right) \sim \frac{d z_{1} d z_{2}}{\left(z_{1}-z_{2}\right)^{2}}+\text { regular . }
$$

$B$ is called the Bergman kernel of $\mathcal{C}$, or the fundamental 2 -form of the second kind [34].

\subsubsection{Recursion kernel}

We now define the recursion kernel $K$ as

$$
K\left(z_{0}, z\right)=\frac{\int_{\bar{z}}^{z} B\left(z_{0}, z^{\prime}\right)}{2(y(\bar{z})-y(z)) d x(z)} .
$$

This kernel is a globally defined 1 -form in the variable $z_{0} \in \mathcal{C}$. In the variable $z$, it is the inverse of a 1-form (that means we have to multiply it with a quadratic differential before computing any integral with it); it is defined only locally near branch points of $x$, such that $K\left(z_{0}, \bar{z}\right)=K\left(z_{0}, z\right)$. At the branch points, it has simple poles,

$$
K\left(z_{0}, z\right) \sim-\frac{B\left(z_{0}, z\right)}{2 d x(z) d y(z)}+\text { regular. }
$$




\subsubsection{Topological recursion}

Correlation forms $W_{n}^{(g)}\left(z_{1}, \ldots, z_{n}\right)$ (not to be confused with the resolvents $W_{i}(z)$ introduced above) are symmetric $n$-forms defined by

$$
\begin{gathered}
W_{1}^{(0)}(z)=-y(z) d x(z), \\
W_{2}^{(0)}\left(z_{1}, z_{2}\right)=B\left(z_{1}, z_{2}\right),
\end{gathered}
$$

and then by recursion (we write collectively $J=\left\{z_{1}, \ldots, z_{n}\right\}$ ),

$$
\begin{aligned}
W_{n+1}^{(g)}\left(z_{0}, J\right)= & \sum_{i} \operatorname{Res}_{z \rightarrow a_{i}} K\left(z_{0}, z\right)\left[W_{n+2}^{(g-1)}(z, \bar{z}, J)\right. \\
& \left.+\sum_{h=0}^{g} \sum_{I \subset J}^{\prime} W_{1+|I|}^{(h)}(z, I) W_{1+n-|I|}^{(g-h)}(\bar{z}, J \backslash I)\right]
\end{aligned}
$$

where $\sum_{I}^{\prime}$ is the sum over all subsets of $J$, restricted to $(h, I) \neq(0, \emptyset)$ and $(h, I) \neq$ $(g, J)$.

Although it is not obvious from the definition, the forms $W_{n}^{(g)}$ are symmetric. For $2-$ $2 g-n<0$, they are meromorphic $n$-forms with poles only at branch points. These poles are of degree at most $6 g-4+2 n$, and have vanishing residues.

For the one matrix model, the $W_{n}^{(g)}$ coincide with the $n$-point function of the trace of the resolvent at order $g$ in the topological expansion.

\subsubsection{Symplectic invariants}

Finally, for $g \geq 2$, we define the symplectic invariants $F_{g}$ (also denoted $W_{0}(g)$ in [3]) by

$$
F_{g}(\mathcal{S})=\frac{1}{2-2 g} \sum_{i} \operatorname{Res}_{z \rightarrow a_{i}} \Phi(z) W_{1}^{(g)}(z),
$$

where $\Phi$ is any function defined locally near branch points of $x$ such that $d \Phi=y d x$.

The definitions of $F_{1}$ and $F_{0}$ are more involved and we refer the reader to $[3]$. $F_{0}$ is called the prepotential, and $F_{1}$ is closely related to the determinant of the Laplacian on $\mathcal{C}$ with metrics $|y d x|^{2}$, see [35, 36].

The $F_{g}(\mathcal{S})$ 's depend only on the orbit of $\mathcal{S}$ under the group of transformations generated by

$\mathfrak{R}: \mathcal{S} \mapsto \tilde{\mathcal{S}}=(\mathcal{C}, x, y+R(x))$ where $R(x)$ is any rational function of $x$,

$\mathfrak{F}: \mathcal{S} \mapsto \tilde{\mathcal{S}}=\left(\mathcal{C}, f(x), y / f^{\prime}(x)\right)$ where $f(x)$ is an analytical function of $x$, with $f^{\prime}$ rational, such that $d f=f^{\prime} d x$ has the same number of zeroes as $d x$,

$\mathfrak{S}: \mathcal{S} \mapsto \tilde{\mathcal{S}}=(\mathcal{C}, y,-x)$. 
These transformations are symplectic, i.e. they leave $d x \wedge d y$ invariant.

The symplectic invariants are homogeneous of degree $2-2 g$,

$$
F_{g}(\mathcal{C}, x, \lambda y)=\lambda^{2-2 g} F_{g}(\mathcal{C}, x, y)
$$

In particular, they are invariant under the parity transformation $F_{g}(\mathcal{C}, x,-y)=F_{g}(\mathcal{C}, x, y)$.

\section{The spectral curve for the topological string's ma- trix model}

Applying the procedure outlined in section 4.2.2 to our matrix model, we will determine a spectral curve $\mathcal{S}_{M M}\left(\mathfrak{X}_{0}\right)$ in this section. [27] demonstrated that for a chain of matrices, we have

$$
\ln Z=\sum_{g} g_{s}^{2 g-2} F_{g}\left(\mathcal{S}_{\mathrm{MM}}\right)
$$

with $F_{g}$ the symplectic invariants of [3]. In our case, since we have engineered our matrix model to yield ${ }^{5} G W_{g}\left(\mathfrak{X}_{0}\right)$ as its partition function, re-computing the partition function via the methods of [27] will yield

$$
G W_{g}\left(\mathfrak{X}_{0}\right)=F_{g}\left(\mathcal{S}_{\mathrm{MM}}\right)
$$

This relation is already quite interesting, as it allows for explicit computation of the Gromov-Witten invariants. Our goal however will be to go further. We will argue that $\mathcal{S}_{\mathrm{MM}}$ is symplectically equivalent to the mirror spectral curve $\mathcal{S}_{\hat{\mathfrak{x}}_{0}}$ of section 2.2 ,

$$
\mathcal{S}_{\mathrm{MM}} \sim \mathcal{S}_{\mathfrak{X}_{0}}
$$

Since the $F_{g}$ 's are symplectic invariants, this will imply the BKMP conjecture for $\mathfrak{X}_{0}$, i.e.

$$
G W_{g}\left(\mathfrak{X}_{0}\right)=F_{g}\left(\mathcal{S}_{\mathfrak{X}_{0}}\right)
$$

\subsection{Applying the chain of matrices rules}

We now apply the rules of section 4.2 .2 to the chain of matrices model introduced in section 3 ,

- Recall that the integration ensembles for the matrices $M_{0}$ and $M_{m+1}$ are such that for each matrix, all eigenvalues are integrated on the same contour (A.7). Hence, $k_{0}=k_{m+1}=1$, and the corresponding filling fractions are equal to $N$. For $i=$ $1, \ldots, m$, the matrix $M_{i}$ is integrated on $H\left(\gamma_{0, i}^{d} \times \gamma_{2, i}^{d} \times \cdots \times \gamma_{n, i}^{d}\right)$, where $\gamma_{j, i}$ is a

\footnotetext{
${ }^{5}$ As we have here reserved the notation $F_{g}$ for the symplectic invariants of our matrix model, we refer to the topological string free energies as $G W_{g}$.
} 
contour which surrounds all points of the form $q^{a_{j, i}+\mathbb{N}}$. There are thus $k_{i}=n+1$ filling fractions, each equal to $d$. The matrices $R_{i}$ are integrated on $H\left(\mathbb{R}_{+}^{N}\right)$. We denote the number of their cuts by $\tilde{k}_{i}$. Hence, $\tilde{k}_{i}=1$, with the respective filling fraction equal to $N$.

According to condition 1 of section 4.2 .2 , the genus of the spectral curve $\mathcal{C}$ is thus given by

$$
\mathfrak{g}=\sum_{i=0}^{m+1}\left(k_{i}-1\right)+\sum_{i=1}^{m+1}\left(\tilde{k}_{i}-1\right)=n m .
$$

- Following condition 2 of section 4.2.2, we introduce functions $x_{i}(z), i=0, \ldots, m+1$, associated to the matrices $M_{i}$, and functions $y_{i}(z), i=1, \ldots, m+1$, associated to the matrices $R_{i}$, as well as two additional functions $y_{0}(z)$ and $y_{m+2}(z)$ at the ends of the chain.

They must satisfy the following requirements:

- Since there is no potential for the matrices $R_{i}$, equation (4.4) implies that we have, for $i=1, \ldots, m+1$,

$$
x_{i}(z)-x_{i-1}(z)=0 .
$$

We can hence suppress the index $i$ on these functions, $x(z)=x_{i}(z)$.

- For $i=1, \ldots, m$, equation (4.4) gives

$$
y_{i}(z)-y_{i+1}(z)=2 V_{\vec{a}_{i}}^{\prime}(x(z))-V_{\vec{a}_{i+1}}^{\prime}(x(z))-V_{\vec{a}_{i-1}}^{\prime}(x(z))-g_{s} \frac{f_{i}^{\prime}(x(z))}{f_{i}(x(z))}-\frac{g_{s} S_{i}+i \pi}{x(z)}
$$

and

$$
\begin{gathered}
y_{0}(z)-y_{1}(z)=V_{\vec{a}_{0}}^{\prime}(x(z))-V_{\vec{a}_{1}}^{\prime}(x(z))-g_{s} \frac{f_{0}^{\prime}(x(z))}{f_{0}(x(z))}, \\
y_{m+1}(z)-y_{m+2}(z)=V_{\vec{a}_{m+1}}^{\prime}(x(z))-V_{\vec{a}_{m}}^{\prime}(x(z))-g_{s} \frac{f_{m+1}^{\prime}(x(z))}{f_{m+1}(x(z))} .
\end{gathered}
$$

More explicitly, in terms of the function

$$
\psi_{q}(x)=x g^{\prime}(x) / g(x)
$$

whose small $g_{s}$ expansion

$$
\begin{aligned}
\psi_{q}(x) & =-\frac{1}{\ln q} \sum_{n=0}^{\infty} \frac{(-1)^{n} B_{n}}{n !}(\ln q)^{n} \operatorname{Li}_{1-\mathrm{n}}(1 / \mathrm{x}) \\
& =\frac{1}{\ln q}\left[\ln \left(1-\frac{1}{x}\right)-\frac{\ln q}{2(x-1)}-\sum_{n=1}^{\infty} \frac{B_{2 n}}{(2 n) !}(\ln q)^{2 n} \operatorname{Li}_{1-2 \mathrm{n}}(\mathrm{x})\right] .
\end{aligned}
$$


we worked out in appendix A of [1], we obtain

$$
\begin{aligned}
& x(z)\left(y_{i+1}(z)-y_{i}(z)\right) \\
& =i \pi+g_{s} S_{i}-g_{s} \sum_{j}\left(2 \psi_{q}\left(q^{a_{j, i}} / x(z)\right)-\psi_{q}\left(q^{a_{j, i+1}} / x(z)\right)-\psi_{q}\left(q^{a_{j, i-1}} / x(z)\right)\right) \\
& \quad+g_{s} \frac{x(z) f_{i}^{\prime}(x(z))}{f_{i}(x(z))},
\end{aligned}
$$

as well as

$$
\begin{gathered}
x(z)\left(y_{1}(z)-y_{0}(z)\right)=-g_{s} \sum_{j} \psi_{q}\left(q^{a_{j, 0}} / x(z)\right)+g_{s} \sum_{j} \psi_{q}\left(q^{a_{j, 1}} / x(z)\right) \\
-g_{s} \sum_{j} \sum_{k=0}^{d-1} \frac{x(z)}{x(z)-q^{a_{j, 0}+k}}, \\
x(z)\left(y_{m+2}(z)-y_{m+1}(z)\right)=-g_{s} \sum_{j} \psi_{q}\left(q^{a_{j, m+1}} / x(z)\right)+g_{s} \sum_{j} \psi_{q}\left(q^{a_{j, m}} / x(z)\right) \\
\quad-g_{s} \sum_{j} \sum_{k=0}^{d-1} \frac{x(z)}{x(z)-q^{a_{j, m+1}+k}}
\end{gathered}
$$

Note that we have explicitly used the fact that the partitions $\alpha_{j, m+1}$ and $\alpha_{j, 0}$ are chosen to be trivial.

- Since the integral over $R_{i}$ is over $H_{N}\left(\mathbb{R}_{+}\right)$, i.e. its eigenvalues are integrated on $\mathbb{R}_{+}$, the integration contour has an endpoint (hard edge) at $y_{i}=0$. Condition 3 hence requires that at a pre-image $y_{i}^{-1}(0)$, which we will refer to as $\infty_{i}$, the following holds

$$
y_{i}\left(\infty_{i}\right)=0, \quad d y_{i}\left(\infty_{i}\right)=0, \quad x(z) \text { has a simple pole at } z=\infty_{i} .
$$

Furthermore, introducing a local parameter $z$ in the neighborhood of $\infty_{i}$, the above translates into

$$
y_{i}(z) \sim z^{2}, \quad x(z) \sim 1 / z .
$$

Hence, $\forall i=1, \ldots, m+1$,

$$
y_{i} \sim \mathcal{O}\left(1 / x^{2}\right)
$$

- The relations (5.1) imply that near $\infty_{i}$, we have

$$
x\left(y_{j+1}-y_{j}\right) \underset{z \rightarrow \infty_{i}}{\sim} i \pi+g_{s} S_{j}+g_{s} \sum_{l=0}^{n}\left(2 a_{l, j}-a_{l, j+1}-a_{l, j-1}\right)+O(1 / x) .
$$

In particular, it follows that $\infty_{j} \neq \infty_{i}$. Thus, all points $\left\{\infty_{1}, \ldots, \infty_{m+1}\right\} \subset x^{-1}(\infty)$ are distinct, i.e. condition 3 requires that $x^{-1}(\infty)$ have at least $m+1$ points. 


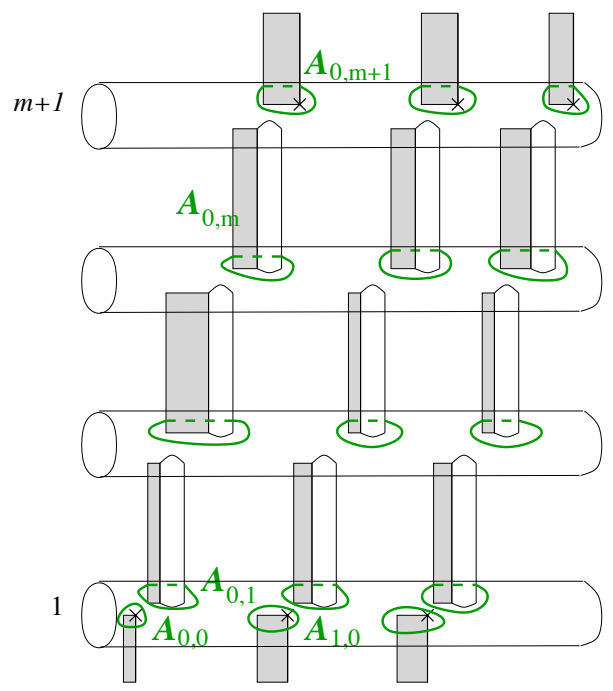

Figure 7: The spectral curve of our matrix model can be represented as follows. The cover of $\mathbb{C P}^{1}$ provided by $x$ has $m+1$ sheets. Instead of the projective plane of $x$, we represent the sheets of $\ln x$, which are cylinders. Cycles $\mathcal{A}_{j, i}$ appear in sheets $i-1$ and $i$. They enclose singularities of the resolvent $W_{i}$. Algebraic cuts are represented as vertical cylinders, and poles and log singularities are represented as grey strips. There is only one cycle $\mathcal{A}_{j, 0}$ (which is in sheet 0 ) and one $\mathcal{A}_{j, m+1}$ (in sheet $m$ ), and they enclose only poles or log singularities of $y_{0}$ resp. $y_{m+1}$.

We will make the minimal assumption that $x^{-1}(\infty)$ has exactly $m+1$ elements that are simple poles of $x$, and that $x$ has no further singularities, i.e. that $x$ is a meromorphic function of degree $m+1$ on $\mathcal{C}$.

- By condition 5, since for $i=0, \ldots, m+2$ there are $d$ eigenvalues of $M_{i}$ of the form $q^{a_{j, i}+\mathbb{N}}$ surrounded by the path $\widehat{\mathcal{A}}_{j, i}$, we have the $(m+2) \times(n+1)$ filling fraction conditions

$$
\frac{1}{2 i \pi} \oint_{\mathcal{A}_{j, i}} y_{i} d x=d g_{s} \quad \text { for } i=0, \ldots, m+1, j=0, \ldots, n .
$$

$x$ hence defines an $m+1$ sheeted cover of $\mathbb{C P}^{1}$. Considering the function $\ln x$ instead, with singularities at $x=0$ and $x=\infty$, each sheet of this cover is mapped to a cylinder. We have depicted this covering in figure 7, and indicated the singularities of $y_{i}$ on each sheet: algebraic cuts are represented by vertical cylinders, and poles and logarithmic cuts by grey strips.

In sheet $i$ we have represented some contours $\mathcal{A}_{j, i}$ whose image under the projection $x: \mathcal{C} \rightarrow \mathbb{C P}^{1}$ surrounds all points of type $q^{a_{j, i}+\mathbb{N}}$.

For $i=1, \ldots, m$, the resolvent $W_{i}(x)$ of the $i^{\text {th }}$ matrix $M_{i}$ is computed as a contour integral around the sum over $j$ of cycles $\mathcal{A}_{j, i}$ on sheet $i$,

$$
W_{i}(x)=\sum_{j=0}^{n} \frac{1}{2 i \pi} \oint_{\mathcal{A}_{j, i}} \frac{y_{i}\left(z^{\prime}\right) d x\left(z^{\prime}\right)}{x-x\left(z^{\prime}\right)} .
$$


Also, as argued in [1], the potentials of $M_{0}$ and $M_{m+1}$ are such that in fact the matrices $M_{0}$ and $M_{m+1}$ are frozen, and thus their resolvents contain only poles. In terms of the functions $y_{0}$ and $y_{m+1}$, we conclude that the singularities of $y_{0}$ in $\mathcal{A}_{j, 0}$ in sheet 1 and the singularities of $y_{m+1}$ in $\mathcal{A}_{j, m+1}$ in sheet $m+1$ can be only poles, not cuts.

Since condition 1 requires that the genus be $\mathfrak{g}=n \mathrm{~m}$, we see that there can be no other cuts than the ones already discussed - the genus would be higher, otherwise.

\subsection{Symplectic change of functions}

The spectral curve of the matrix model is $\mathcal{S}_{M M}=\left(\mathcal{C}, x, y_{0}\right)$, and our goal is to relate it to the mirror curve described in section 2.2. The mirror curve is described via the algebraic equation 2.3) in the two functions $x_{1}, x_{2}: \mathcal{C}_{\text {mirror }} \rightarrow \mathbb{C P}^{1}$ (in the patch $x_{0}=1$ ). We wish to obtain a similar algebraic description of $\mathcal{C}$. Due to $\log$ singularities in $y_{0}$, to be traced to the small $g_{s}$ behavior of $\psi_{q}(x)$, an algebraic equation in the variables $\left(x, y_{0}\right)$ cannot exist (recall that $x$ is meromorphic). In this section, we shall, via a series of symplectic transformations on the $y_{i}$ of the type enumerated in section 4.3.5, arrive at functions $Y_{i}$ that are meromorphic on $\mathcal{C}$, and hence each present a viable candidate to pair with $x$ to yield an algebraic equation for $\mathcal{C}$.

Essentially, we wish to introduce the exponentials of $y_{i}$. While this will eliminate the log singularities, poles in $y_{i}$ would be elevated to essential singularities. We hence first turn to the question of eliminating these poles.

\subsubsection{The arctic circle property}

On the physical sheet, the interpretation of a pole of $y_{i}$ is as an eigenvalue of the matrix $M_{i}$ with delta function support. Such a so-called frozen eigenvalue can arise in the following way:

The sum over all partitions is dominated by partitions close to a typical equilibrium partition, i.e. a saddle point. The typical partition has a certain typical length referred to as its equilibrium length $\bar{n}$. All partitions with a length very different from the equilibrium length contribute only in an exponentially small way (and thus non-perturbatively) to the full partition function. Introducing a cutoff on the length of partitions which is larger than the equilibrium length hence does not change the perturbative part of the partition function. Now recall that when we defined the $h_{i}(\gamma)$ of a representation $\gamma$ in appendix A, we introduced an arbitrary maximal length $d$ such that $l(\gamma) \leq d$ and set

$$
h_{i}(\gamma)=a_{\gamma}+d-i+\gamma_{i}
$$

Setting $\gamma_{i}=0$ for $d \geq i>\bar{n}$ yields $h_{i}$ that do not depend on the integration variables, hence are frozen at fixed values. This behavior is referred to as the arctic circle property [37], as all eigenvalues beyond the arctic circle situated at equilibrium length $\bar{n}$ are frozen. 
Returning to our matrix model, the eigenvalues of the matrices $M_{i}$ are given by $q^{\left(h_{j, i}\right)_{l}}$. For $d \geq l>n_{j, i}$, they are frozen, and thus contribute poles to $y_{i}$ by (4.5) (recall that poles of the resolvent correspond to eigenvalues with delta function support) in the physical sheet. We will assume that these are the only poles in the physical sheet and we subtract them to obtain new functions $\tilde{y}_{i}$,

$$
\begin{gathered}
\tilde{y}_{0}(z)=x(z) y_{0}(z)-\sum_{j} \sum_{k=0}^{d-1} \frac{g_{s} x(z)}{x(z)-q^{a_{j, 0}+k}}, \\
\tilde{y}_{m+2}(z)=x(z) y_{m+2}(z)+\sum_{j} \sum_{0}^{d-1} \frac{g_{s} x(z)}{x(z)-q^{a_{j, m+1}+k}}
\end{gathered}
$$

and for $i=1, \ldots, m+1$,

$$
\tilde{y}_{i}(z)=x(z) y_{i}(z)-\sum_{j} \sum_{k=0}^{d-n_{j, i}-1} \frac{g_{s} x(z)}{x(z)-q^{a_{j, i}+k}}+\sum_{j} \sum_{k=0}^{d-n_{j, i-1}-1} \frac{g_{s} x(z)}{x(z)-q^{a_{j, i-1}+k}} .
$$

We have set

$$
n_{j, 0}=0, \quad n_{j, m+1}=0 .
$$

Notice that at large $x(z)$ in sheet $i$ we have

$$
\tilde{y}_{0} \sim O(1 / x(z)), \quad \tilde{y}_{m+2} \sim O(1 / x(z))
$$

and for $i=1, \ldots, m+1$

$$
\tilde{y}_{i} \sim g_{s} \sum_{j}\left(n_{j, i}-n_{j, i-1}\right)+O(1 / x(z)) .
$$

As a general property of $\psi_{q}$, we have for any integer $n_{j, i} \leq d$

$$
\psi_{q}\left(q^{a_{j, i}} / x\right)=\psi_{q}\left(q^{a_{j, i}+d-n_{j, i}} / x\right)+\sum_{k=0}^{d-n_{j, i}-1} \frac{x}{x-q^{a_{j, i}+k}} .
$$

Hence, the loop equations for the new functions $\tilde{y}_{i}$ read

$$
\begin{aligned}
\tilde{y}_{i+1}(z)-\tilde{y}_{i}(z)= & i \pi+g_{s} S_{i} \\
& +g_{s} \sum_{j}\left(2 \psi_{q}\left(q^{a_{j, i}+d-n_{j, i}} / x(z)\right)-\psi_{q}\left(q^{a_{j, i+1}+d-n_{j, i+1}} / x(z)\right)\right. \\
& \left.-\psi_{q}\left(q^{a_{j, i-1}+d-n_{j, i-1}} / x(z)\right)\right)+g_{s} \frac{x(z) f_{i}^{\prime}(x(z))}{f_{i}(x(z))}, \\
\tilde{y}_{1}(z)-\tilde{y}_{0}(z)= & g_{s} \sum_{j} \psi_{q}\left(q^{a_{j, 0}+d} / x(z)\right)-g_{s} \sum_{j} \psi_{q}\left(q^{a_{j, 1}+d-n_{j, 1}} / x(z)\right),
\end{aligned}
$$



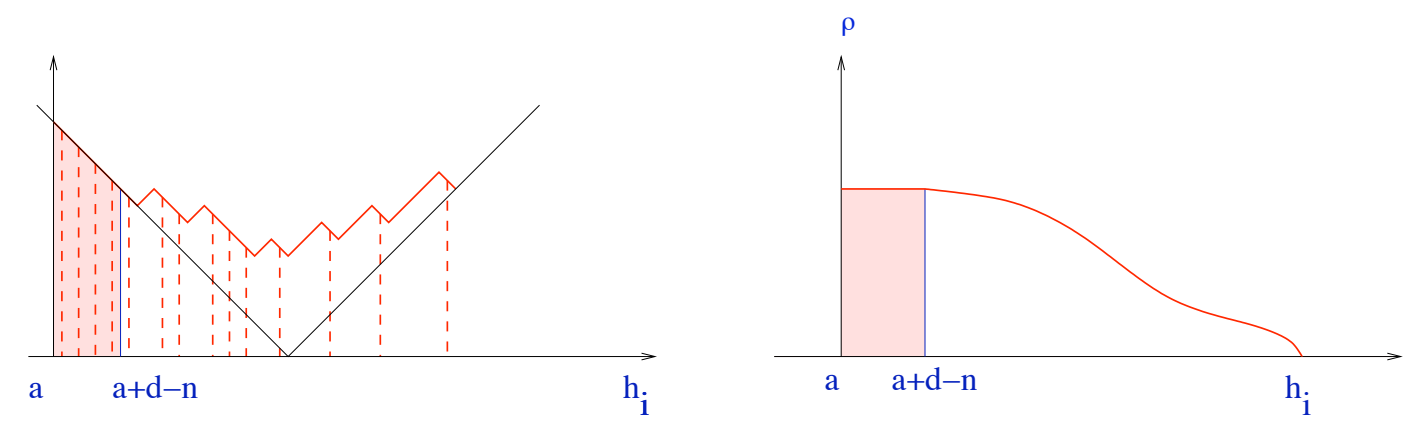

Figure 8: We shift the cut-off $d$ on the representation lengths, $d \rightarrow n_{j, i}$, with $n_{j, i}$ chosen such that frozen eigenvalues in the expected distribution of the $h_{i}$ are suppressed. In the limit of vanishing spacing $\left(g_{s} \rightarrow 0\right)$, the equidistant frozen eigenvalues give rise to a constant eigenvalue density region.

$$
\tilde{y}_{m+2}(z)-\tilde{y}_{m+1}(z)=g_{s} \sum_{j} \psi_{q}\left(q^{a_{j, m+1}+d} / x(z)\right)-g_{s} \sum_{j} \psi_{q}\left(q^{a_{j, m}+d-n_{j, m}} / x(z)\right) .
$$

The $n_{j, i}$ in the above definitions are defined as the equilibrium lengths, i.e. by the property that the functions $\tilde{y}_{i}$ have no poles on their physical sheet. That such a choice of $n_{j, i}$ exists is suggested by the arctic circle property.

Note that the $n_{j, i}$ can also be specified by the fact that $q^{a_{j, i}+d-n_{j, i}}$ be the beginning of the cut encircled by $\gamma_{j, i}$. As we have identified the discontinuities of $y_{i}$ to lie across branchcuts of $x$, this implies that $x$ has ramification points at the element of $x^{-1}\left(q^{a_{j, i}+d-n_{j, i}}\right)$ lying on the physical sheets of $y_{i}$.

Note that the arctic circle property also implies the perturbative independence of our expressions from the arbitrary cut-off $d$. Changing $d$ to $d+d^{\prime}$ merely introduces $d^{\prime}$ new frozen eigenvalues $h_{i}$. This independence from $d$ is important in establishing the equality between the topological string partition function and our matrix integral (3.1), as the topological vertex formulae in fact are formulated in the limit $d \rightarrow \infty$.

\subsubsection{Obtaining globally meromorphic functions}

We have arrived at functions $\tilde{y}_{i}$ that have no poles on their physical sheet, and are thus safely exponentiated there. We wish now to use the loop equations to obtain functions which are globally well-behaved.

To this end, we note that since the Gromov-Witten invariants are defined as a formal power series in $g_{s}$, we can compute the spectral curve order by order in $g_{s}$, invoking the following small $\ln q$ expansion [1]:

$$
\begin{aligned}
\psi_{q}\left(q^{a_{j, i}+d-n_{j, i}} / x\right) \sim & -\frac{1}{g_{s}} \ln \left(1-\frac{x}{q^{a_{j, i}+d-n_{j, i}}}\right)+\frac{x}{2\left(x-q^{a_{j, i}+d-n_{j, i}}\right)} \\
& +\frac{1}{g_{s}} \sum_{n=1}^{\infty} \frac{B_{2 n} g_{s}^{2 n}}{(2 n) !} \operatorname{Li}_{1-2 \mathrm{n}}\left(\mathrm{q}^{\mathrm{d}-\mathrm{n}_{\mathrm{j}, \mathrm{i}}+\mathrm{a}_{\mathrm{j}, \mathrm{i}} / \mathrm{x}}\right) .
\end{aligned}
$$


The functions $f_{i}^{\prime} / f_{i}$ are completely non-perturbative; one can easily check with the above expansion that they can be replaced by 0 to every order in $g_{s}$.

Introducing new functions $X(z)$ and $Y_{i}$ by the formulae

$$
\begin{aligned}
x(z) & =q^{d} X(z), \\
\tilde{y}_{0}(z) & =\ln Y_{0}(z), \\
\tilde{y}_{m+2}(z) & =\ln Y_{m+2}(z),
\end{aligned}
$$

and for $i=1, \ldots, m+1$

$$
\begin{aligned}
\tilde{y}_{i}(z)= & \ln Y_{i}(z)+\sum_{j} \frac{X(z) g_{s}}{2\left(X(z)-q^{a_{j, i}-n_{j, i}}\right)}+\frac{1}{g_{s}} \sum_{j} \sum_{n=1}^{\infty} \frac{B_{2 n} g_{s}^{2 n}}{(2 n) !} \operatorname{Li}_{1-2 \mathrm{n}}\left(\mathrm{q}^{\mathrm{a}_{\mathrm{j}, \mathrm{i}}-\mathrm{n}_{\mathrm{j}, \mathrm{i}}} / \mathrm{X}(\mathrm{z})\right) \\
& -\sum_{j} \frac{X(z) g_{s}}{2\left(X(z)-q^{\left.a_{j, i-1}-n_{j, i-1}\right)}\right.}-\frac{1}{g_{s}} \sum_{j} \sum_{n=1}^{\infty} \frac{B_{2 n} g_{s}^{2 n}}{(2 n) !} \operatorname{Li}_{1-2 \mathrm{n}}\left(\mathrm{q}^{\mathrm{a}_{\mathrm{j}, \mathrm{i}-1}-\mathrm{n}_{\mathrm{j}, \mathrm{i}-1}} / \mathrm{X}(\mathrm{z})\right)
\end{aligned}
$$

yields loop equations that are algebraic on their right hand side,

$$
\begin{aligned}
& \frac{Y_{i}}{Y_{i+1}}=-e^{-g_{s} S_{i}} \prod_{j} \frac{\left(X-q^{a_{j, i+1}-n_{j, i+1}}\right)\left(X-q^{a_{j, i-1}-n_{j, i-1}}\right)}{\left(X-q^{a_{j, i}-n_{j, i}}\right)^{2}} \\
& \prod_{j} q^{2\left(a_{j, i}-n_{j, i}\right)-\left(a_{j, i+1}-n_{j, i+1}\right)-\left(a_{j, i-1}-n_{j, i-1}\right)}, \\
& \frac{Y_{0}}{Y_{1}}=\prod_{j} \frac{\left(X-q^{a_{j, 1}-n_{j, 1}}\right)}{\left(X-q^{a_{j, 0}}\right)} \prod_{j} q^{a_{j, 0}-\left(a_{j, 1}-n_{j, 1}\right)}, \\
& \frac{Y_{m+1}}{Y_{m+2}}=\prod_{j} \frac{\left(X-q^{a_{j, m}-n_{j, m}}\right)}{\left(X-q^{a_{j, m+1}}\right)} \prod_{j} q^{a_{j, m+1}-\left(a_{j, m}-n_{j, m}\right)},
\end{aligned}
$$

i.e.

$$
\frac{Y_{i}}{Y_{0}}=e^{g_{s}\left(S_{1}+\cdots+S_{i-1}\right)} \prod_{j} q^{\left(a_{j, i}-n_{j, i}\right)-\left(a_{j, i-1}-n_{j, i-1}\right)} \prod_{j} \frac{X-q^{a_{j, i-1}-n_{j, i-1}}}{X-q^{a_{j, i}-n_{j, i}}}
$$

Since we have argued that the $Y_{i}$ are holomorphic on their physical sheet, and the ratio $Y_{i} / Y_{i+1}$ is purely algebraic, we conclude that the $Y_{i}$ are meromorphic functions on all of $\mathcal{C}$. This was the goal we had set out to achieve.

Note that the above changes of variables have modified the asymptotics at infinity and the integrals over the $\mathcal{A}$-cycles. More precisely, we have

$$
\begin{gathered}
\forall i \in[1, m+1]: \ln Y_{i} \underset{\infty_{i}}{\sim} \tilde{y}_{i} \underset{\infty_{i}}{\sim} g_{s} \sum_{j}\left(n_{j, i}-n_{j, i-1}\right)+O\left(\frac{1}{x}\right), \\
\ln Y_{0}=\tilde{y}_{0} \underset{\infty}{\sim} O\left(\frac{1}{x}\right),
\end{gathered}
$$




$$
\ln Y_{m+2}=\tilde{y}_{m+2} \underset{\infty}{\sim} O\left(\frac{1}{x}\right) .
$$

The filling fraction equation reads

$$
\frac{1}{2 i \pi} \int_{\mathcal{A}_{j, i}} \frac{\tilde{y}_{i}(z)}{x(z)} d x(z)=g_{s}\left(d-\left(d-n_{j, i}\right)\right)=g_{s} n_{j, i} .
$$

In terms of $Y_{0}$, these conditions can be rewritten as

$$
\ln Y_{0} \underset{\infty_{i}}{\sim}-g_{s}\left(S_{1}+\cdots+S_{i-1}\right)+g_{s} \sum_{j=0}^{n}\left(a_{j, i}-a_{j, i-1}\right)+O\left(\frac{1}{x}\right)
$$

and

$$
\begin{aligned}
\frac{1}{2 i \pi} \int_{\mathcal{A}_{j, i}} \ln Y_{0} \frac{d X}{X} & =\frac{1}{2 i \pi} \int_{\mathcal{A}_{j, i}} \ln Y_{i} \frac{d X}{X}+\frac{1}{2 i \pi} \int_{\mathcal{A}_{j, i}} \ln X d \ln \left(\frac{Y_{i}}{Y_{0}}\right) \\
& =\frac{1}{2 i \pi} \int_{\mathcal{A}_{j, i}} \ln Y_{i} \frac{d X}{X}+\frac{1}{2 i \pi} \int_{\mathcal{A}_{j, i}} \ln X d\left(\sum_{k=1}^{i} \ln \frac{Y_{k}}{Y_{k-1}}\right) \\
& =\frac{1}{2 i \pi} \int_{\mathcal{A}_{j, i}} \ln Y_{i} \frac{d X}{X}-\frac{1}{2 i \pi} \int_{\mathcal{A}_{j, i}} \ln X\left(\sum_{l} \frac{d X}{X-q^{a_{l, i}-n_{l, i}}}-\frac{d X}{X-q^{a_{l, i-1}-n_{l, i-1}}}\right) \\
& =\frac{1}{2 i \pi} \int_{\mathcal{A}_{j, i}} \ln Y_{i} \frac{d X}{X}+g_{s}\left(a_{j, i}-n_{j, i}\right) \\
& =g_{s} n_{j, i}+g_{s}\left(a_{j, i}-n_{j, i}\right) \\
& =g_{s} a_{j, i} .
\end{aligned}
$$

\subsection{Recovering the mirror curve}

We have argued above that $X$ and $Y_{i}$, and hence in particular $Y_{0}$, are meromorphic functions on $\mathcal{C}$. There must hence exist a polynomial $H(X, Y)$ such that (see e.g. Theorem 5.8 .1 in [38])

$$
H\left(X, Y_{0}\right)=0 .
$$

The facts that $X$ provides an $m+1$ sheeted cover of $\mathbb{C P}^{1}$ and that $Y_{0}$ may have $n+1$ poles in its physical sheet imply that the polynomial $H$ has degrees at least $(n+1, m+1)$. As above, we shall choose the minimal hypothesis that it has exactly these degrees. Thus,

$$
H(X, Y)=\sum_{i=0}^{m+1} \sum_{j=0}^{n+1} H_{i, j} X^{j} Y^{i}
$$

As we saw in section 2.2, projectivizing a generic polynomial of these degrees (yielding a homogeneous polynomial of degree $m+n+2$ ) indeed gives rise to a curve of genus $\mathfrak{g}=n m$.

We now need to determine the $(n+2)(m+2)-1$ unknown coefficients of $H$ ( $H$ is defined up to a global multiplicative constant). 
The cycle integrals

$$
\oint_{\mathcal{A}_{j, i}} \ln Y_{0} \frac{d X}{X}=2 i \pi g_{s} a_{j, i}
$$

provide $(n+1) m$ constraints on the coefficients of $H$. We also have $m+1$ constraints for the behavior at $\infty_{i}, i=1, \ldots, m+1$,

$$
\operatorname{Res}_{\infty_{i}} \ln Y_{0} \frac{d X}{X}=g_{s}\left(S_{1}+\cdots+S_{i-1}\right)-g_{s} \sum_{j=0}^{n}\left(a_{j, i}-a_{j, i-1}\right) .
$$

Finally, requiring that $Y_{0}$ has poles at $q^{a_{j, 0}}$ and $Y_{m+2}$ has zeroes at $q^{a_{j, m+1}}$ gives another $2(n+1)$ constraints, which we may write as

$$
\begin{gathered}
\underset{q^{a_{j j, 0}}}{\operatorname{Res}} \ln X \frac{d Y_{0}}{Y_{0}}=g_{s} a_{j, 0}, \\
\underset{q^{a_{j, m+1}}}{\operatorname{Res}} \ln X \frac{d Y_{m+2}}{Y_{m+2}}=g_{s} a_{j, m+1} .
\end{gathered}
$$

This gives enough equations to completely determine $H$. Knowing $H$, we know the location of branch points as functions of $a_{j, i}$ 's and $S_{i}$ 's, and can hence determine the $n_{j, i}$ by requiring that $q^{a_{j, i}-n_{j, i}}$ be a branch point.

Notice that we can choose to express the period integrals in any linear combination of $\mathcal{A}$-cycles. In particular,

$$
\begin{gathered}
\oint_{\mathcal{A}_{j, i+1}-\mathcal{A}_{j, i}} \ln Y_{0} \frac{d X}{X}=2 i \pi g_{s}\left(a_{j, i+1}-a_{j, i}\right)=2 i \pi g_{s} t_{j, i}, \\
\oint_{\mathcal{A}_{j, i+1}-\mathcal{A}_{j+1, i}} \ln Y_{0} \frac{d X}{X}=2 i \pi g_{s}\left(a_{j, i+1}-a_{j+1, i}\right)=2 i \pi g_{s} r_{j, i} .
\end{gathered}
$$

Similarly, we may also take linear combinations of $\mathcal{A}$-cycles together with circles surrounding the poles or zeroes of $x$ in order to get the $s_{j, i}$ classes. We hence conclude that the periods of the curve $H\left(X, Y_{0}\right)=0$ yield the quantum corrected Kähler parameters of the fiducial toric geometry $\mathfrak{X}_{0}$, allowing us to identify it with the corresponding mirror curve.

\subsection{Topological expansion and symplectic invariants}

Following [27], we obtained

$$
\mathcal{S}_{M M}=\left(\mathcal{C}, x, y_{0}\right)
$$

as the spectral curve of our matrix model at the end of section 5.1 .

As reviewed in section 4.3 , we can compute the corresponding symplectic invariants $F_{g}\left(\check{\mathcal{S}}_{M M}\right)$, which assemble to yield the matrix model partition function [27],

$$
\ln Z=\sum_{g} g_{s}^{2 g-2} F_{g}\left(\mathcal{S}_{M M}\right) .
$$




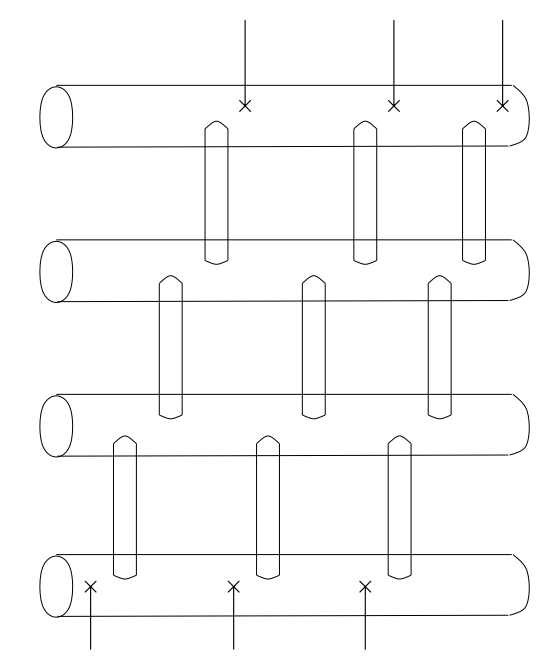

Figure 9: The spectral curve $\left(X, \frac{1}{X} \ln \left(Y_{0}\right)\right)$ has the following structure: $X(z)$ is a meromorphic function of degree $m+1$ on a curve of genus $\mathfrak{g}=n m$. Therefore it has $m+1$ poles and $m+1$ zeroes. It provides a branched covering of $\mathbb{C P}^{1}$. We prefer to represent $\ln X$ instead of $X$, and thus we have $m+1$ copies of the $\ln X$-cylinder. In each sheet there is one zero and one pole of $X . Y_{0}$ is a meromorphic function of degree $n+1$, so that it has $n+1$ zeroes in sheet 0 , and $n+1$ poles in sheet $m+1$. We recognize the mirror curve $\mathcal{S}_{\hat{\mathfrak{X}}_{0}}$, which is a thickening of the toric web diagram.

The symplectic transformation $\mathfrak{R}$ of section 4.3 .5 maps $\left(\mathcal{C}, x, y_{0}\right)$ to $\left(\mathcal{C}, x, \frac{1}{x} \ln Y_{0}\right)$ order by order in $g_{s} . \mathfrak{F}$ maps this to $\left(\mathcal{C}, X, \frac{1}{X} \ln Y_{0}\right)$, and a second application of $\mathfrak{F}$ yields

$$
\hat{\mathcal{S}}_{M M}=\left(\mathcal{C}, \ln X, \ln Y_{0}\right) .
$$

By the symplectic invariance of the $F_{g}$, we therefore have, order by order in powers of $g_{s}$,

$$
F_{g}\left(\mathcal{S}_{M M}\right)=F_{g}\left(\hat{\mathcal{S}}_{M M}\right) .
$$

Since our matrix model was engineered to reproduce the Gromov-Witten invariants of $\mathfrak{X}_{0}$, we have arrived at

$$
G W_{g}\left(\mathfrak{X}_{0}\right)=F_{g}\left(\mathcal{C}, \ln X, \ln Y_{0}\right),
$$

with $X$ and $Y_{0}$ obeying the algebraic equation

$$
H_{0}\left(X, Y_{0}\right)=0
$$

which coincides with the equation (2.3) describing the mirror curve of $\mathfrak{X}_{0}$.

Given our minimality assumptions on the spectral curve, we have thus derived the BKMP conjecture for the fiducial geometry $\mathfrak{X}_{0}$.

\subsection{The small $q$ limit and the thickening prescription}

The above derivation of the spectral curve for the matrix model is not fully rigorous, as we have relied on making minimal assumptions along the way. Although the spectral 
curve we have found here satisfies all the constraints of section 4.2 .2 , to prove that it is the spectral curve of our matrix model requires a uniqueness result which we currently do not have.

In this section, we provide a heuristic argument that the qualitative behavior of the spectral curve and the mirror curve coincide at small $q$.

At small $q$, only very small partitions contribute to the matrix integral. Almost all eigenvalues of $M_{i}$ are frozen to the values $q^{a_{j, i}+d-l}$. By the arguments in section 5.2.1, the resolvent $W_{i}(x)$ hence behaves at small $q$ as

$$
W_{i}(x) \sim \sum_{j=0}^{n} \sum_{l=1}^{d} \frac{1}{x-q^{a_{j, i}-l+d}}+\text { small cut near } q^{a_{j, i}+d} .
$$

Pictorially, the size of the cuts is shrinking in this limit, replacing the spectral curve by its skeleton, see figure 10 .

On the other hand, the mirror curve is a priori a tree level quantity, hence does not depend on $q=e^{-g_{s}}$. However, recall that we have defined the Kähler parameters $Q$ associated to a curve $\mathcal{C}$ as

$$
Q=q^{\int_{\mathcal{C}} J}
$$

The large $q$ limit hence corresponds to the large curve class limit, i.e. the distance between the vertices of the pairs of pants out of which the mirror curve is constructed is taken to infinity. Just as the spectral curve, the mirror curve thus collapses to its skeleton in the $q \rightarrow 0$ limit.

\section{The general BKMP conjecture}

So far, we have obtained the BKMP conjecture only for the fiducial geometry $\mathfrak{X}_{0}$. Studying the behavior of the partition function under flop transitions will allow us to extend our argument to arbitrary toric geometries.

\subsection{Flop invariance of toric Gromov-Witten invariants}

Under the proper identification of curve classes, Gromov-Witten invariants (at least on toric manifolds) are invariant under flops. Assume the toric Calabi-Yau manifolds $\mathfrak{X}$ and $\mathfrak{X}^{+}$are related via a flop transition, $\phi: \mathfrak{X} \rightarrow \mathfrak{X}^{+}$. In a neighborhood of the flopped $(-1,-1)$ curve, the respective toric diagrams are depicted in figure 11 .

The 1-cones of $\Sigma_{\mathfrak{X}}$, corresponding to the toric invariant divisors of $\mathfrak{X}$, are not affected by the flop, hence can be canonically identified with those of $\mathfrak{X}^{+}$. The 2-cones $\tau_{i}$ in these diagrams correspond to toric invariant 2-cycles $C_{i}, C_{i}^{+}$in the geometry. The curve classes of $\mathfrak{X}$ push forward to classes in $\mathfrak{X}^{+}$via

$$
\phi_{*}\left(\left[C_{0}\right]\right)=-\left[C_{0}^{+}\right], \quad \phi_{*}\left(\left[C_{i}\right]\right)=\left[C_{i}^{+}\right]+\left[C_{0}^{+}\right] .
$$




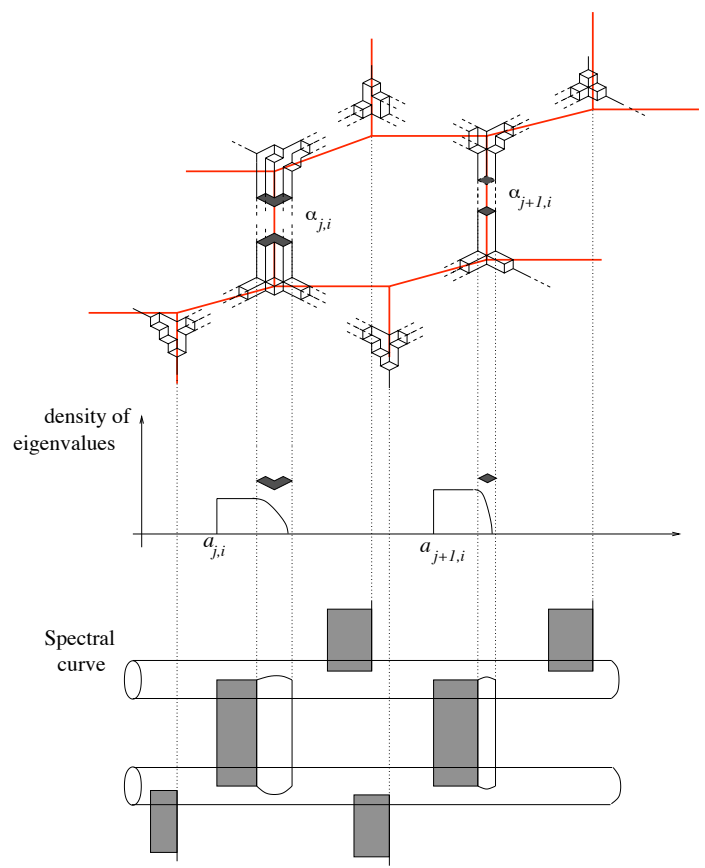

Figure 10: In the small $q$ limit, only very small partitions contribute to the matrix integral, therefore the density of eigenvalues of $M_{i}$ tends to the flat density (a Dirac comb of equidistant delta functions), the non-flat part, which reflects the cuts of the spectral cut, shrinks to zero.
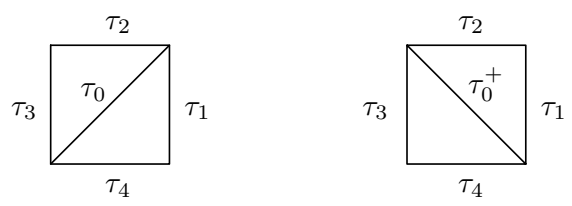

Figure 11: $\mathfrak{X}$ and $\mathfrak{X}^{+}$in the vicinity of the $(-1,-1)$ curve.

All other curve classes $\vec{C}$ of $\mathfrak{X}$ are mapped to their canonical counterparts in $\mathfrak{X}^{+}$. Under appropriate analytic continuation and up to a phase factor (hence the $\propto$ in the following formula), the following identity then holds [39, 23, 40],

$$
Z_{G W}\left(\mathfrak{X}, Q_{0}, Q_{1}, \ldots, Q_{4}, \vec{Q}\right) \propto Z_{G W}\left(\mathfrak{X}^{+}, 1 / Q_{0}, Q_{0} Q_{1}, \ldots, Q_{0} Q_{4}, \vec{Q}\right),
$$

i.e.

$$
G W_{g}\left(\mathfrak{X}, Q_{0}, Q_{1}, \ldots, Q_{4}, \vec{Q}\right)=G W_{g}\left(\mathfrak{X}^{+}, 1 / Q_{0}, Q_{0} Q_{1}, \ldots, Q_{0} Q_{4}, \vec{Q}\right) .
$$

\subsection{Proof of flop invariance via mirror symmetry}

Flop invariance of Gromov-Witten invariants upon the identification (6.1) is immediate upon invoking mirror symmetry, as (6.1) maps the mirror curve of $\mathfrak{X}$ to that of $\mathfrak{X}^{+}$. The proof is a simple computation. 

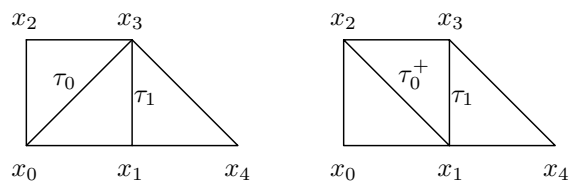

Figure 12 : $\mathfrak{X}$ and $\mathfrak{X}^{+}$in the vicinity of the $(-1,-1)$ curve.

Let us introduce the notation $t_{0}, t_{1}, t_{0}^{+}, t_{1}^{+}$for the Kähler volume of the curve classes $C_{i}, C_{i}^{+}$ corresponding to the respective 2-cones. In terms of these, we obtain for the mirror curve of $\mathfrak{X}$

$$
x_{0}+x_{1}+x_{2}+\frac{x_{1} x_{2}}{x_{0}} e^{T_{0}}+\frac{x_{1}^{2}}{x_{0}} e^{-T_{1}}=0,
$$

while the mirror curve of $\mathfrak{X}^{+}$is given by

$$
x_{0}+x_{1}+x_{2}+\frac{x_{1} x_{2}}{x_{0}} e^{-T_{0}^{+}}+\frac{x_{1} x_{3}}{x_{2}} e^{-T_{1}^{+}}=0 .
$$

Upon invoking $x_{3}=\frac{x_{1} x_{2}}{x_{0}} e^{-T_{0}^{+}}$, we easily verify that the identification 6.1 maps these curves and their associated meromorphic 1-forms $\lambda$ into each other.

\subsection{The BKMP conjecture}

Any toric Calabi-Yau manifold $\mathfrak{X}$ with Kähler moduli $\vec{Q}$ can be obtained from a sufficiently large fiducial geometry $\left(\mathfrak{X}_{0}, \vec{Q}_{0}\right)$ upon performing a series of flop transitions and taking unwanted Kähler moduli of $\mathfrak{X}_{0}$ to $\infty$, see figure 13 for an example.

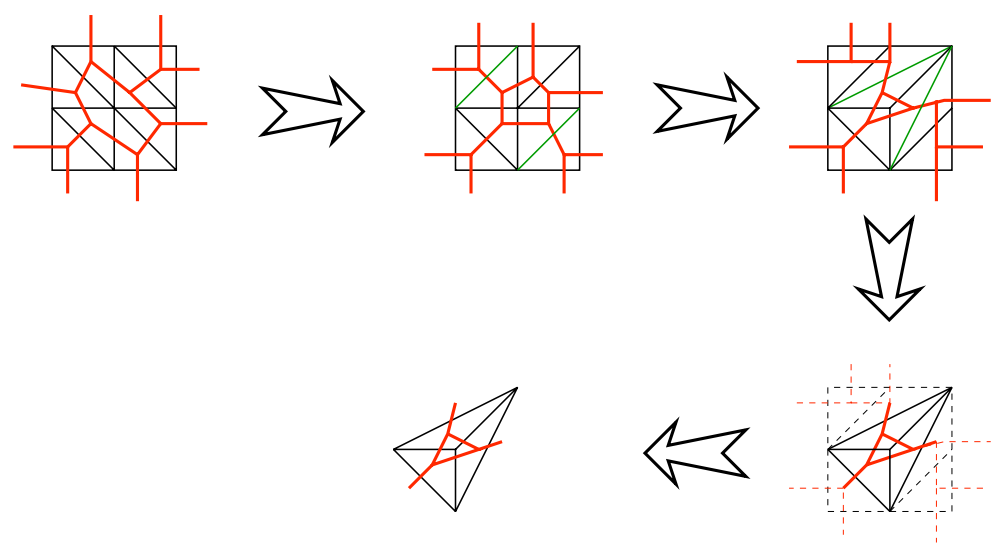

Figure 13: Example: We obtain local $\mathbb{P}^{2}$ from the fiducial geometry with $2 \times 2$ boxes by performing five flops and then sending the Kähler parameters of the unwanted edges to $\infty$.

The Kähler moduli of $\mathfrak{X}$ are related to those of $\mathfrak{X}_{0}$ by some relation $\vec{Q}=f\left(\vec{Q}_{0}\right)$. We have just argued that the mirror curves of $\mathfrak{X}_{0}$ and $\mathfrak{X}$ are equal upon this identification,

$$
\mathcal{S}_{\mathfrak{X}, \vec{Q}}=\mathcal{S}_{\mathfrak{X}_{0}, \overrightarrow{Q_{0}}},
$$


as are the respective Gromov-Witten invariants,

$$
G W_{g}(\mathfrak{X}, \vec{Q})=G W_{g}\left(\mathfrak{X}_{0}, \vec{Q}_{0}\right) .
$$

Given the BKMP conjecture for the fiducial geometry,

$$
G W_{g}\left(\mathfrak{X}_{0}, \vec{Q}_{0}\right)=F_{g}\left(\mathcal{S}_{\mathfrak{X}_{0}, \vec{Q}_{0}}\right),
$$

its validity thus follows for any toric Calabi-Yau manifold:

$$
G W_{g}(\mathfrak{X}, \vec{Q})=F_{g}\left(\mathcal{S}_{\mathfrak{X}, \vec{Q}}\right)
$$

\section{Conclusion}

Taking our matrix model from [1] as a starting point and imposing certain minimality conditions on the spectral curve, we have thus derived the BKMP conjecture, for closed topological strings, for all toric Calabi-Yau manifolds in the large radius limit. As we have emphasized throughout, elevating our procedure to a formal proof of the conjecture requires a more rigorous derivation of the spectral curve of our matrix model.

It should also be possible to extend our argument to open Gromov-Witten invariants by invoking loop operators, which relate closed to open invariants. In [3], such an operator was defined in the matrix model context. An analogous operator should also exist in the theory of Gromov-Witten invariants [41]. Establishing the equivalence of these two loop operators would allow us to conclude that the $W_{n}^{(g)}$ 's of the spectral curve $\mathcal{S}_{\mathfrak{X}}$ are the open Gromov-Witten invariants of $\mathfrak{X}$.

Finally, our treatment of the BKMP conjecture took place at large radius. One should study the behavior of the matrix model as one moves away from large radius e.g. to orbifold points, and see whether the phase transitions of the topological string are captured accurately by the matrix model. Of course, the main tool on the topological string side employed in this work, the topological vertex, is no longer applicable in these regions of moduli space.

\section{Acknowledgments}

B.E. and O.M. would like to thank M. Bertola, J. Harnad, V. Bouchard, M. Mariño, M. Mulase, H. Ooguri, N. Orantin, B. Safnuk, for useful and fruitful discussions on this subject. A.K. would like to thank Vincent Bouchard and Ilarion Melnikov for helpful conversations. The work of B.E. is partly supported by the Enigma European network MRT-CT-2004-5652, ANR project GranMa "Grandes Matrices Aléatoires" ANR08-BLAN-0311-01, by the European Science Foundation through the Misgam program, 
by the Quebec government with the FQRNT. B.E. would like to thank the AIM, as well as the organizers and all participants to the workshop [42] held at the AIM june 2009. O.M. would like to thank the CRM (Centre de recheche mathématiques de Montréal, QC, Canada) for its hospitality.

\section{A The matrix model}

In this appendix, which is mainly a reprint of section 4 of [1], we present the matrix model which reproduces the topological string partition function on the fiducial geometry $\mathfrak{X}_{0}$, and whose spectral curve we derive in the text.

SConsider the fiducial geometry $\mathfrak{X}_{0}$ of size $(n+1) \times(m+1)$, with Kähler parameters $t_{i, j}=a_{i, j}-a_{i, j+1}, r_{i, j}=a_{i, j+1}-a_{i+1, j}$, and $s_{i, j}$, as depicted in figure 1. We write

$$
\vec{a}_{i}=\left(a_{0, i}, a_{1, i}, \ldots, a_{n, i}\right)
$$

Assume that the external representations are fixed to $\vec{\alpha}_{m+1}=\left(\alpha_{0, m+1}, \alpha_{1, m+1}, \ldots, \alpha_{n, m+1}\right)$ on the upper line, and $\vec{\alpha}_{0}=\left(\alpha_{0,0}, \alpha_{1,0}, \ldots, \alpha_{n, 0}\right)$ on the lower line. For the most part, we will choose these to be trivial.

We now define the following matrix integral $\mathcal{Z}_{\mathrm{MM}}$ (MM for Matrix Model),

$$
\begin{aligned}
\mathcal{Z}_{\mathrm{MM}}\left(Q, g_{s}, \vec{\alpha}_{m+1}, \vec{\alpha}_{0}^{T}\right)= & \Delta\left(X\left(\vec{\alpha}_{m+1}\right)\right) \Delta\left(X\left(\vec{\alpha}_{0}\right)\right) \prod_{i=0}^{m+1} \int_{H_{N}\left(\Gamma_{i}\right)} d M_{i} \prod_{i=1}^{m+1} \int_{H_{N}\left(\mathbb{R}_{+}\right)} d R_{i} \\
& \prod_{i=1}^{m} e^{\frac{-1}{g_{s}} \operatorname{tr}\left[V_{\vec{a}_{i}}\left(M_{i}\right)-V_{\vec{a}_{i-1}}\left(M_{i}\right)\right]} \prod_{i=1}^{m} e^{\frac{-1}{g_{s}} \operatorname{tr}\left[V_{\vec{a}_{i-1}}\left(M_{i-1}\right)-V_{\vec{a}_{i}}\left(M_{i-1}\right)\right]} \\
& \prod_{i=1}^{m+1} e^{\frac{1}{g_{s}} \operatorname{tr}\left(M_{i}-M_{i-1}\right) R_{i}} \prod_{i=1}^{m} e^{\left(S_{i}+\frac{i \pi}{g_{s}}\right) \operatorname{tr} \ln M_{i}} \\
& e^{\operatorname{tr} \ln f_{0}\left(M_{0}\right)} e^{\operatorname{tr} \ln f_{m+1}\left(M_{m+1}\right)} \prod_{i=1}^{m} e^{\operatorname{tr} \ln f_{i}\left(M_{i}\right)}
\end{aligned}
$$

All matrices are taken of size

$$
N=(n+1) d .
$$

$d$ denotes a cut-off on the size of the matrices, on which, as discussed in section 5.2.1, the partition function depends only non-perturbatively. We have introduced the notation

$$
\begin{aligned}
X\left(\vec{\alpha}_{m+1}\right)=\operatorname{diag}\left(X\left(\vec{\alpha}_{m+1}\right)_{i}\right)_{i=1, \ldots, N}, & X\left(\vec{\alpha}_{m+1}\right)_{j d+k}=q^{h_{k}\left(\alpha_{j, m+1}\right)} \\
X\left(\vec{\alpha}_{0}\right)=\operatorname{diag}\left(X\left(\vec{\alpha}_{0}\right)_{i}\right)_{i=1, \ldots, N}, & X\left(\vec{\alpha}_{0}\right)_{j d+k}=q^{h_{k}\left(\alpha_{j, 0}\right)}
\end{aligned}
$$

for $k=1, \ldots, d, j=0, \ldots, n$, where

$$
h_{i}(\gamma)=\gamma_{i}-i+d+a
$$


$\Delta(X)=\prod_{i<j}\left(X_{i}-X_{j}\right)$ is the Vandermonde determinant. The potentials $V_{\vec{a}_{i}}(x)$ are given by

$$
V_{\vec{a}}(X)=-g_{s} \sum_{j=0}^{n} \ln \left(g\left(q^{a_{j}} / X\right)\right)
$$

in terms of the $q$-product

$$
g(x)=\prod_{n=1}^{\infty}\left(1-\frac{1}{x} q^{n}\right) .
$$

For $i=1, \ldots, m$, we have defined

$$
f_{i}(x)=\prod_{j=0}^{n} \frac{g(1)^{2} e^{\left(\frac{1}{2}+\frac{i \pi}{\ln q}\right) \ln \left(x q^{1-a_{j, i}}\right)} e^{\frac{\left(\ln \left(x q^{1-a_{j, i}}\right)\right)^{2}}{2 g_{s}}}}{g\left(x q^{1-a_{j, i}}\right) g\left(q^{a_{j, i}} / x\right)} .
$$

The denominator of these functions induces simple poles at $x=q^{a_{j, i}+l}$ for $j=0, \ldots, n$ and $l \in \mathbb{Z}$. The numerator is chosen such that they satisfy the relation $f_{i}(q x)=f_{i}(x)$. This enforces a simple $l$-dependence of the residues taken at $x=q^{a_{j, i}+l}$, given by a prefactor $q^{l}$ - a fact which will be important in the following. These residues are in fact given by

$$
\operatorname{Res}_{q^{a_{j, i}+l}} f_{i}(x)=q^{a_{j, i}+l} \hat{f}_{j, i}=-q^{a_{j, i}+l} \prod_{k \neq j} \frac{g(1)^{2} e^{\left(\frac{1}{2}+\frac{i \pi}{\ln q}\right)\left(1+a_{j, i}-a_{k, i}\right) \ln q} e^{\frac{\left(\operatorname { l n } \left(q^{\left.\left.1+a_{j, i}-a_{k, i}\right)\right)^{2}}\right.\right.}{2 g_{s}}}}{g\left(q^{a_{j, i}-a_{k, i}}\right)\left(1-q^{a_{k, i}-a_{j, i}}\right) g\left(q^{a_{k, i}-a_{j, i}}\right)},
$$

where $\hat{f}_{j, i}$ is independent of the integer $l$.

The parameters $S_{i}$ are defined by

$$
S_{i}=s_{0, i-1}+t_{0, i-1}=s_{j, i-1}-\sum_{k<j} t_{k, i}+\sum_{k \leq j} t_{k, i-1}
$$

The final equality holds for arbitrary $j[1]$.

For $i=0$ and $i=m+1$, we define

$$
\begin{aligned}
f_{0}(x) & =\frac{1}{\prod_{j=0}^{n} \prod_{i=1}^{d}\left(x-q^{h_{i}\left(\alpha_{j, 0}\right)}\right)}, \\
f_{m+1}(x) & =\frac{1}{\prod_{j=0}^{n} \prod_{i=1}^{d}\left(x-q^{h_{i}\left(\alpha_{j, m+1}\right)}\right)} .
\end{aligned}
$$

Notice that if the representations $\vec{\alpha}_{0}$ or $\vec{\alpha}_{m+1}$ are trivial, i.e. $h_{i}\left(\alpha_{j, 0}\right)=d-i+a_{j, 0}$ or $h_{i}\left(\alpha_{j, m+1}\right)=d-i+a_{j, m+1}$, we have

$$
f_{0}(x)=\prod_{j=0}^{n} \frac{g\left(x q^{1-a_{j, 0}-d}\right)}{x^{d} g\left(x q^{1-a_{j, 0}}\right)}, \quad f_{m+1}(x)=\prod_{j=0}^{n} \frac{g\left(x q^{1-a_{j, m+1}-d}\right)}{x^{d} g\left(x q^{1-a_{j, m+1}}\right)}
$$


respectively. The functions $f_{0}$ and $f_{m+1}$ have simple poles at $x=q^{h_{l}\left(\alpha_{j, 0}\right)}$ (resp. $x=$ $\left.q^{h_{l}\left(\alpha_{j, m+1}\right)}\right)$ for $l=1, \ldots, d$, with residue

$$
\begin{gathered}
\hat{f}_{j, 0 ; l}=\operatorname{Res}_{q^{h_{l}\left(\alpha_{j, 0}\right)}} f_{0}(x)=\frac{1}{\prod_{j^{\prime} \neq j} \prod_{i=1}^{d}\left(q^{h_{l}\left(\alpha_{j, 0}\right)}-q^{h_{i}\left(\alpha_{j^{\prime}, 0}\right)}\right)} \frac{1}{\prod_{i \neq l}\left(q^{h_{l}\left(\alpha_{j, 0}\right)}-q^{h_{i}\left(\alpha_{j, 0}\right)}\right)}, \\
\hat{f}_{j, m+1 ; l}=\operatorname{Res}_{q^{h_{l}\left(\alpha_{j, m+1}\right)}} f_{m+1}(x)=\frac{1}{\prod_{j^{\prime} \neq j} \prod_{i=1}^{d}\left(q^{h_{l}\left(\alpha_{j, m+1}\right)}-q^{h_{i}\left(\alpha_{j^{\prime}, m+1}\right)}\right)} \frac{1}{\prod_{i \neq l}\left(q^{h_{l}\left(\alpha_{j, m+1}\right)}-q^{h_{i}\left(\alpha_{j, m+1}\right)}\right)} .
\end{gathered}
$$

The $l$ dependence here is more intricate than above, but this will not play any role since the partitions $\alpha_{j, 0}$ and $\alpha_{j, m+1}$ are kept fixed, and not summed upon.

The integration domains for the matrices $R_{i}$ are $H_{N}\left(\mathbb{R}_{+}^{N}\right)$, i.e. the set of hermitian matrices having only positive eigenvalues. For the matrices $M_{i}, i=1, \ldots, m$, the integration domains are $H_{N}\left(\Gamma_{i}\right)$, where

$$
\Gamma_{i}=\prod_{j=0}^{n}\left(\gamma_{j, i}\right)^{d}
$$

$\gamma_{j, i}$ is defined as a contour which encloses all points of the form $q^{a_{j, i}+\mathbb{N}}$, and does not intersect any contours $\gamma_{k, l},(j, i) \neq(k, l)$. For this to be possible, we must require that the differences $a_{j, i}-a_{j^{\prime}, i^{\prime}}$ be non-integer. The normalized logarithms of two such contours are depicted in figure 14 .

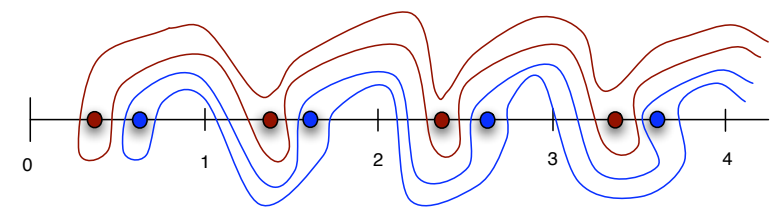

Figure 14: Two contours surrounding points $a+\mathbb{N}$ and $b+\mathbb{N}$, such that $a-b \notin \mathbb{Z}$.

We have defined

$$
H_{N}\left(\Gamma_{i}\right)=\left\{M=U \Lambda U^{\dagger}, \quad U \in U(N), \Lambda=\operatorname{diag}\left(\lambda_{1}, \ldots, \lambda_{N}\right) \in \Gamma_{i}\right\},
$$

i.e. $H_{N}\left(\Gamma_{i}\right)$ is the set of normal matrices with eigenvalues on $\Gamma_{i}$. By definition, the measure on $H_{N}\left(\Gamma_{i}\right)$ is (see [24])

$$
d M=\frac{1}{N !} \Delta(\Lambda)^{2} d U d \Lambda
$$

where $d U$ is the Haar measure on $U(N)$, and $d \Lambda$ is the product of the measures for each eigenvalue along its integration path.

The integration domains for the matrices $M_{0}, M_{m+1}$ are $H_{N}\left(\Gamma_{0}\right), H_{N}\left(\Gamma_{m+1}\right)$ respectively, where

$$
\Gamma_{0}=\left(\sum_{j=0}^{n} \gamma_{j, 0}\right)^{N}, \quad \Gamma_{m+1}=\left(\sum_{j=0}^{n} \gamma_{j, m+1}\right)^{N}
$$




\section{References}

[1] B. Eynard, A.-K. Kashani-Poor, and O. Marchal, "A matrix model for the topological string I: Deriving the matrix model," arXiv:1003.1737 [hep-th].

[2] B. Eynard, "Topological expansion for the 1-hermitian matrix model correlation functions," JHEP 11 (2004) 031, arXiv:hep-th/0407261.

[3] B. Eynard and N. Orantin, "Invariants of algebraic curves and topological expansion," arXiv:math-ph/0702045.

[4] B. Eynard, M. Marino, and N. Orantin, "Holomorphic anomaly and matrix models," JHEP 06 (2007) 058, arXiv:hep-th/0702110.

[5] N. Orantin, "Symplectic invariants, Virasoro constraints and Givental decomposition," arXiv:0808.0635 [math-ph].

[6] L. Chekhov, A. Marshakov, A. Mironov, and D. Vasiliev, "DV and WDVV," Phys. Lett. B562 (2003) 323-338, arXiv: hep-th/0301071.

[7] B. Eynard, "Recursion between Mumford volumes of moduli spaces," arXiv:0706.4403.

[8] V. Bouchard, A. Klemm, M. Marino, and S. Pasquetti, "Remodeling the B-model," Commun. Math. Phys. 287 (2009) 117-178, arXiv:0709.1453 [hep-th].

[9] M. Marino, "Open string amplitudes and large order behavior in topological string theory," JHEP 03 (2008) 060, arXiv:hep-th/0612127.

[10] B. Eynard, "All orders asymptotic expansion of large partitions," J. Stat. Mech. 0807 (2008) P07023, arXiv:0804.0381 [math-ph].

[11] A. Marshakov and N. Nekrasov, "Extended Seiberg-Witten theory and integrable hierarchy," JHEP 01 (2007) 104, arXiv: hep-th/0612019.

[12] A. Klemm and P. Sulkowski, "Seiberg-Witten theory and matrix models," Nucl. Phys. B819 (2009) 400-430, arXiv:0810.4944 [hep-th].

[13] P. Sulkowski, "Matrix models for $2^{*}$ theories," arXiv:0904.3064 [hep-th].

[14] J. Zhou, "Local Mirror Symmetry for One-Legged Topological Vertex," (2009) , arXiv:0910.4320 [math.AG].

[15] L. Chen, "Bouchard-Klemm-Marino-Pasquetti Conjecture for $\mathbb{C}^{3}$," (2009), arXiv:0910.3739 [math.AG].

[16] V. Bouchard and M. Marino, "Hurwitz numbers, matrix models and enumerative geometry," arXiv:0709.1458 [math.AG].

[17] G. Borot, B. Eynard, M. Mulase, and B. Safnuk, "A matrix model for simple Hurwitz numbers, and topological recursion," arXiv:0906.1206 [math-ph].

[18] B. Eynard, M. Mulase, and B. Safnuk, "The Laplace transform of the cut-and-join equation and the Bouchard-Marino conjecture on Hurwitz numbers," arXiv:0907.5224 [math.AG].

[19] H. Ooguri, P. Sulkowski, and M. Yamazaki, "Wall Crossing As Seen By Matrix 
Models," arXiv:1005.1293 [hep-th].

[20] K. Hori and C. Vafa, "Mirror symmetry," arXiv:hep-th/0002222.

[21] M. Aganagic, A. Klemm, and C. Vafa, "Disk instantons, mirror symmetry and the duality web," Z. Naturforsch. A57 (2002) 1-28, arXiv:hep-th/0105045.

[22] M. Aganagic, A. Klemm, M. Marino, and C. Vafa, "The topological vertex," Commun. Math. Phys. 254 (2005) 425-478, arXiv:hep-th/0305132.

[23] A. Iqbal and A.-K. Kashani-Poor, "The vertex on a strip," Adv. Theor. Math. Phys. 10 (2006) 317-343, arXiv:hep-th/0410174.

[24] M. L. Mehta, Random matrices, vol. 142 of Pure and Applied Mathematics (Amsterdam). Elsevier/Academic Press, Amsterdam, third ed., 2004.

[25] P. Di Francesco, P. H. Ginsparg, and J. Zinn-Justin, "2-D Gravity and random matrices," Phys. Rept. 254 (1995) 1-133, arXiv:hep-th/9306153

[26] B. Eynard, "Master loop equations, free energy and correlations for the chain of matrices," JHEP 11 (2003) 018, arXiv:hep-th/0309036.

[27] B. Eynard and A. P. Ferrer, "Topological expansion of the chain of matrices," JHEP 07 (2009) 096.

[28] B. Eynard and O. Marchal. work in progress.

[29] M. Bertola, "Biorthogonal polynomials for 2-matrix models with semiclassical potentials," J.APPROX.THEORY 1442 (2007) 162.

[30] B. Eynard and N. Orantin, "Algebraic methods in random matrices and enumerative geometry," arXiv:0811.3531 [math-ph].

[31] G. Bonnet, F. David, and B. Eynard, "Breakdown of universality in multi-cut matrix models," J. Phys. A33 (2000) 6739-6768, arXiv: cond-mat/0003324.

[32] B. Eynard, "Loop equations for the semiclassical 2-matrix model with hard edges," J. Stat. Mech. 0510 (2005) P006, arXiv:math-ph/0504002.

[33] L. Chekhov, "Matrix models with hard walls: Geometry and solutions," J.Phys.A 39 (2006) 8857-8894, arXiv:0602013 [hep-th]

[34] J. D. Fay, Theta functions on Riemann surfaces. Lecture Notes in Mathematics, Vol. 352. Springer-Verlag, Berlin, 1973.

[35] A. Kokotov and D. Korotkin, "Tau-function on Hurwitz spaces," Mathematical Physics, Analysis and Geometry 7 (2004) 47-96.

[36] B. Eynard, A. Kokotov, and D. Korotkin, "Genus one contribution to free energy in hermitian two- matrix model," Nucl. Phys. B694 (2004) 443-472, arXiv:hep-th/0403072.

[37] K. Johansson, "The arctic circle boundary and the Airy process," Ann. Probab. 33 (2005) no. 1, 1-30.

[38] J. Jost, Compact Riemann surfaces. Springer-Verlag, Berlin, second ed., 2002. An introduction to contemporary mathematics.

[39] E. Witten, "Phases of $\mathrm{N}=2$ theories in two dimensions," Nucl. Phys. B403 (1993) 
159-222, arXiv:hep-th/9301042.

[40] Y. Konishi and S. Minabe, "Flop invariance of the topological vertex," Int. J. Math. 19 (2008) 27-45, arXiv:math/0601352.

[41] R. Cavalieri. private communications.

[42] Recursion structures in topological string theory and enumerative geometry. June 8 to June 12, 2009, at the American Institute of Mathematics (AIM), Palo Alto, California. 